\title{
SEMI-CLASSICAL BOUNDS ON SCATTERING CROSS SECTIONS IN TWO DIMENSIONAL MAGNETIC FIELDS
}

\author{
HIDEO TAMURA
}

\begin{abstract}
We prove the uniform boundedness of averaged total cross sections or of quantities related to scattering into cones in the semi-classical limit for scattering by two dimensional magnetic fields. We do not necessarily assume that the energy under consideration is in a non-trapping energy range in the sense of classical dynamics.
\end{abstract}

\section{$\S 1$. Introduction}

The present work is a continuation to [20] where we have studied the shadow scattering (the quantum total cross section doubles the classical one in the semi-classical limit) in magnetic fields under the assumption that the energy under consideration is in a non-trapping energy range in the sense of classical dynamics and we have proved that the shadow scattering is in gerenal violated in the case of scattering by magnetic fields. We here study the problem about the uniform boundedness of averaged total cross sections or of quantities related to scattering into cones in the semi-classical limit without assuming such a non-trapping energy condition. In final section (Section 9), we also study the bound on cross sections for scattering by magnetic fields with small support. As a conclusion, we can obtain that such a bound seems to depend on the flux of magnetic fields.

Throughout the whole exposition, we work exclusively in the two dimensional space $R^{2}$ with generic point $x=\left(x_{1}, x_{2}\right)$. Let $A(x)=\left(a_{1}(x)\right.$, $\left.a_{2}(x)\right): R^{2} \rightarrow R^{2}$ be a smooth magnetic vector potential and let

$$
H(A)=(-i \nabla-A)^{2} / 2=\sum_{j=1}^{2}\left(D_{j}-a_{j}\right)^{2} / 2
$$

be the Schrödinger operator associated with magnetic potential $A$, where $D_{j}=-i \partial_{j}=-i \partial / \partial x_{j}$. We sometimes identify $A$ with the one-form $A=$

Received October 4, 1995. 
$a_{1} d x_{1}+a_{2} d x_{2}$ and the magnetic field $b(x)=\partial_{1} a_{2}-\partial_{2} a_{1}$ with the twoform $d A=b(x) d x_{1} \wedge d x_{2}$. Throughout the entire discussion, $b(x)$ is always assumed to satisfy that:

$$
b(x) \in C_{0}^{\infty}\left(R^{2}\right) \text { is a real smooth function with compact support. }
$$

The Hamiltonian $H(A)$ describes a quantum particle of unit mass moving in the magnetic field $b$. The magnetic potential $A(x)$ with $d A=b$ is not uniquely determined and cannot be expected to fall off rapidly at infinity, even if $b(x)$ is assumed to be compactly supported. In fact, $A(x)$ does not decay faster than $O\left(|x|^{-1}\right)$, if

$$
\beta=(2 \pi)^{-1} \int b(x) d x
$$

does not vanish $(2 \pi \beta$ being called the flux of magnetic field $b$ ), where the integral with no domain attached is taken over the whole space. We use this abbreviation throughout. Thus the perturbation $H(A)-H_{0}$ to the free Hamiltonian $H_{0}=-\Delta / 2$ is of long-range class. This implies that the scattering matrix does not necessarily admit the usual decomposition $I d+\{$ Hilbert-Schmidt class $\}, I d$ being the identity operator, as in the shortrange scattering case and also the total cross section is not necessarily finite. Indeed, it depends on the value $\beta$ and becomes finite only for integer $\beta \in Z$.

We shall explain the above matter more precisely. The total cross section is invariant under gauge transformations. We fix one of magnetic potentials $A(x)$ with $d A=b$ and define it as follows:

$$
\begin{aligned}
& a_{1}(x)=-(2 \pi)^{-1} \partial_{2} \int \log |x-y| b(y) d y \\
& a_{2}(x)=(2 \pi)^{-1} \partial_{1} \int \log |x-y| b(y) d y
\end{aligned}
$$

As is easily seen, $A(x)=\left(a_{1}(x), a_{2}(x)\right)$ satisfies $b=d A$ and behaves like

$$
\begin{aligned}
& a_{1}(x)=-\beta x_{2} /|x|^{2}+O\left(|x|^{-2}\right), \\
& a_{2}(x)=\beta x_{1} /|x|^{2}+O\left(|x|^{-2}\right)
\end{aligned}
$$

as $|x| \rightarrow \infty$. Thus $A(x)$ does not fall off faster than $O\left(|x|^{-1}\right)$ at infinity, provided that $\beta \neq 0$.

We denote by $H=H(A)$ the Schrödinger operator with $A(x)$ defined above as a magnetic potential. This operator formally defined by (1.1) 
admits a unique self-adjoint realization in the space $L^{2}\left(R^{2}\right)$ with domain $H^{2}\left(R^{2}\right)$ (Sobolev space). We also denote this realization as the same notation $H=H(A)$. As previously stated, the perturbation $H(A)-H_{0}$ is of long-range class. Nevertheless we know $([11,12])$ that the ordinary wave operators

$$
W_{ \pm}\left(H, H_{0}\right)=s-\lim _{t \rightarrow \pm \infty} \exp (i t H) \exp \left(-i t H_{0}\right)
$$

exist and are asymptotically complete

$$
\operatorname{Ran} W_{ \pm}\left(H, H_{0}\right)=L^{2}\left(R^{2}\right) .
$$

It is also known $([9])$ that $H$ has no bound states. Hence the scattering operator

$$
S\left(H, H_{0}\right)=W_{+}^{*}\left(H, H_{0}\right) W_{-}\left(H, H_{0}\right): L^{2}\left(R^{2}\right) \rightarrow L^{2}\left(R^{2}\right)
$$

can be defined as a unitary operator on $L^{2}\left(R^{2}\right)$ and it has the direct integral decomposition

$$
S\left(H, H_{0}\right)=\int_{0}^{\infty} \oplus S\left(\lambda ; H, H_{0}\right) d \lambda,
$$

where the fiber $S\left(\lambda ; H, H_{0}\right)$ is called a scattering matrix at energy $\lambda$ and acts as a unitary operator on $L^{2}\left(S^{1}\right), S^{1}$ being the unit circle. According to the results due to $[1,10]$, the scattering matrix has an integral kernel $S\left(\theta, \omega, \lambda ; H, H_{0}\right)$ smooth in $\lambda>0$ and $(\theta, \omega) \in S^{1} \times S^{1}, \theta \neq \omega$. However the scattering matrix $S\left(\lambda ; H, H_{0}\right)$ does not necessarily take such a form as $I d+T(\lambda)$ with Hilbert-Schmidt operator $T(\lambda)$ acting on $L^{2}\left(S^{1}\right)$ because of the long-range perturbation. In other words, the scattering kernel $S\left(\theta, \omega, \lambda ; H, H_{0}\right)$ does not necessarily admit the delta-function $\delta(\theta-\omega)$ as a singularity near the forward direction $\theta=\omega$.

We shall analyse such a singularity near the forward direction. We now write

$$
\gamma(x)=\tan ^{-1}\left(x_{2} / x_{1}\right), \quad 0 \leq \gamma<2 \pi,
$$

for the azimuth angle from the positive $x_{1}$ axis. Then

$$
\nabla \gamma=\left(-x_{2} /|x|^{2}, x_{1} /|x|^{2}\right)
$$

and hence it follows from (1.5) that

$$
A(x)=\beta \nabla \gamma+O\left(|x|^{-2}\right), \quad|x| \rightarrow \infty .
$$


It should be noted that $\nabla \gamma$ is smooth in $R^{2} \backslash\{0\}$, although $\gamma(x)$ itself is smooth only in the plane split along the positive $x_{1}$ axis. We also introduce the auxiliary Hamiltonian

$$
H_{\beta}=H(B)=(-i \nabla-B)^{2} / 2, \quad B(x)=\beta \nabla \gamma(x),
$$

for which the perturbation $H-H_{\beta}$ is of short-range class. By the chain rule of wave operators, we obtain

$$
W_{ \pm}\left(H, H_{0}\right)=W_{ \pm}\left(H, H_{\beta}\right) W_{ \pm}\left(H_{\beta}, H_{0}\right)
$$

and hence it follows that

$$
S\left(H, H_{0}\right)=W_{+}^{*}\left(H_{\beta}, H_{0}\right) S\left(H, H_{\beta}\right) W_{-}\left(H_{\beta}, H_{0}\right),
$$

where $S\left(H, H_{\beta}\right)=W_{+}^{*}\left(H, H_{\beta}\right) W_{-}\left(H, H_{\beta}\right)$. The magnetic potential $B(x)$ represents so-called magnetic string and has a strong singularity at the origin. Thus the operator $H_{\beta}$ does not necessarily have the same domain as $H$ or $H_{0}$. However it has been proved in [15] that the wave operators $W_{ \pm}\left(H_{\beta}, H_{0}\right)$ exist and are complete $\operatorname{Ran} W_{ \pm}\left(H_{\beta}, H_{0}\right)=L^{2}\left(R^{2}\right)$. Therefore the existence and completeness of wave operators $W_{ \pm}\left(H, H_{\beta}\right)$ also follow immediately from (1.6) and (1.7). As is easily seen, $H_{\beta}$ is rotationally invariant and hence it admits the partial wave expansion. This enables us to calculate explicitly the scattering kernel of $S\left(\lambda ; H_{\beta}, H_{0}\right)([15])$. It takes the form

$$
\left(S\left(\lambda ; H_{\beta}, H_{0}\right) f\right)(\theta)=\int_{0}^{2 \pi} S_{\beta}\left(\theta-\theta^{\prime}\right) f\left(\theta^{\prime}\right) d \theta^{\prime}, \quad f \in L^{2}\left(S^{1}\right),
$$

where $\theta$ and $\theta^{\prime}$ denote the azimuth angles from the positive $x_{1}$ axis and $S_{\beta}(\theta)$ is given by

$$
S_{\beta}(\theta)=\cos \beta \pi \delta(\theta)-i \pi^{-1} \sin \beta \pi \exp (i m \theta) \text { v.p. } \frac{\exp (i \theta)}{\exp (i \theta)-1}
$$

with $m=[\beta],[]$ being the Gauss notation. Here v.p. stands for the principal value. If, in particular, $\beta$ is an even integer, then $S\left(\lambda ; H_{\beta}, H_{0}\right)=$ $I d$ and also if $\beta$ is an odd integer, then $S\left(\lambda ; H_{\beta}, H_{0}\right)=-I d$. Since $H-$ $H_{\beta}$ is a short-range perturbation as stated above, the scattering matrix $S\left(\lambda ; H, H_{\beta}\right)$ has the decomposition $I d+T(\lambda)$ with Hilbert-Schmidt operator $T(\lambda)$. Thus the operator

(1.10) $T(\lambda ; b)=i(2 \pi)^{-1}\left(S\left(\lambda ; H, H_{0}\right)-S\left(\lambda ; H_{\beta}, H_{0}\right)\right): L^{2}\left(S^{1}\right) \rightarrow L^{2}\left(S^{1}\right)$ 
becomes an integral operator of Hilbert-Schmidt class and it has the kernel

$$
T(\theta, \omega, \lambda ; b)=i(2 \pi)^{-1}\left(S\left(\theta, \omega, \lambda ; H, H_{0}\right)-S\left(\theta, \omega, \lambda ; H_{\beta}, H_{0}\right)\right)
$$

We give the concrete expression for $T(\theta, \omega ; \lambda ; b)$ in the next section (see Lemma 2.1). This is represented in terms of generalized eigenfunctions $\varphi_{ \pm}(x, \lambda, \omega)$ of $H_{\beta}$ and has a definite meaning as a quantity related to scattering involving the pair $\left(H_{\beta}, H\right)$, when $H$ is considered as a short-range perturbation to $H_{\beta}$.

We define the differential cross section for scattering from incident direction $\omega$ to final one $\theta$ at energy $\lambda>0$. It takes the form

$$
|f(\omega \rightarrow \theta, \lambda ; b)|^{2}=2 \pi(2 \lambda)^{-1 / 2}\left|S\left(\theta, \omega, \lambda ; H, H_{0}\right)-\delta(\theta-\omega)\right|^{2}
$$

and the total cross section is defined as

$$
\sigma_{\text {tot }}(\lambda, \omega ; b)=\int|f(\omega \rightarrow \theta, \lambda ; b)|^{2} d \theta .
$$

The above integral is convergent for integer $\beta \in Z$ but is divergent for non-integer $\beta \notin Z$ because of strong singurality near the forward direction. Thus the total cross section $\sigma_{\text {tot }}(\lambda, \omega)$ is finite only for $\beta \in Z$. We now introduce

$$
\sigma(\lambda, \omega ; b)=(2 \pi)^{3}(2 \lambda)^{-1 / 2} \int|T(\theta, \omega, \lambda ; b)|^{2} d \theta
$$

as a basic quantity to be analysed here. This integral is finite even for the case $\beta \notin Z$. If, in particular, $\beta \in Z$, then $\sigma(\lambda, \omega ; b)$ just coincides with the total cross section $\sigma_{\text {tot }}(\lambda, \omega ; b)$.

We move to the the semi-classical case and formulate the main result. Let

$$
H_{0}(h)=-h^{2} \Delta / 2, \quad H(h)=H(h ; A)=(-i h \nabla-A)^{2} / 2, \quad 0<h \ll 1,
$$

where the magnetic potential $A(x)$ is again defined by (1.4). For the pair of semi-classical Hamiltonians $\left(H_{0}(h), H(h)\right)$, the basic quantity $\sigma_{h}(\lambda, \omega ; b)$ is given by

$$
\sigma_{h}(\lambda, \omega ; b)=\sigma(k, \omega ; g b)
$$

with 


$$
g=h^{-1} \gg 1, \quad k=\lambda h^{-2}=\lambda g^{2} .
$$

We average this quantity with respect to incident direction $\omega$ and define

$$
\sigma_{\mathrm{av}, h}(\lambda ; b)=(2 \pi)^{-1} \int \sigma_{h}(\lambda, \omega ; b) d \omega .
$$

Then the main theorem is formulated as follows.

TheOrem 1.1. Let the notations be as above. Assume that the magnetic field $b(x)$ fulfills (1.2). Then one has

$$
\sigma_{\mathrm{av}, h}(\lambda ; b)=O(1), \quad h \rightarrow 0
$$

locally uniformly in $\lambda>0$.

Assume that $\beta / h \in Z$ is an integer. Then the total cross section for the pair $\left(H_{0}(h), H(h)\right)$ is finite and $\sigma_{\text {tot }, h}(\lambda, \omega ; b)=\sigma_{h}(\lambda, \omega ; b)$. We define the averaged total cross section as

$$
\sigma_{\text {avtot }, h}(\lambda ; b)=(2 \pi)^{-1} \int \sigma_{\text {tot }, h}(\lambda, \omega ; b) d \omega .
$$

As an immediate consequence of the above theorem, we obtain the following

Corollary 1.2. Assume that (1.2) is fulfilled. If $\beta / h \in Z$ is an integer, then

$$
\sigma_{\text {avtot }, h}(\lambda ; b)=O(1), \quad h \rightarrow 0,
$$

where $h$ tends to zero with restriction $\beta / h \in Z$. If, in particular, $b=b(|x|)$ is a radial magnetic field, then $\sigma_{\text {tot }, h}(\lambda, \omega ; b)=O(1)$ as $h \rightarrow 0$.

We give another application of Theorem 1.1. Let $g=h^{-1}$ and $k=\lambda h^{-2}$ be as in (1.13). Then the differential cross section for the pair $\left(H_{0}(h), H(h)\right)$ is given by

$$
\left|f_{h}(\omega \rightarrow \theta, \lambda ; b)\right|^{2}=|f(\omega \rightarrow \theta, k ; g b)|^{2} .
$$

Let $\rho(\omega)$ be a bounded function with support in a small neighborhood of some direction $\omega_{0}$ fixed. We consider the following integral

$$
\sigma_{h}(\lambda, \rho, C)=\int_{\Lambda} \int\left|f_{h}(\omega \rightarrow \theta, \lambda ; b)\right|^{2}|\rho(\omega)|^{2}\left(\cos \omega^{\prime}\right)^{-1} d \omega d \theta
$$


where $C=(0, \infty) \times \Lambda \subset R^{2}$ is the cone generated by the compact subset $\Lambda \subset S^{1}$ and $\omega^{\prime}$ denotes the angle between directions $\omega$ and $\omega_{0}$. The above quantity represents the total number of particles scattered into cone $C$ at energy $\lambda$ for the initial state having incident direction $\omega$ subject to probability density $|\rho(\omega)|^{2}([2]$, Chap. 7). If supp $\rho \cap \Lambda=\emptyset$, then it is easily seen that $\sigma_{h}(\lambda, \rho, C)<\infty$ even for the case $\beta / h \notin Z$.

Corollary 1.3. Assume that (1.2) is fulfilled. Let $C=(0, \infty) \times \Lambda$ be the cone generated by the compact subset $\Lambda \subset S^{1}$ and let $\sigma_{h}(\lambda, \rho, C)$ be as above. If supp $\rho \cap \Lambda=\emptyset$, then

$$
\sigma_{h}(\lambda, \rho, C)=O(1), \quad h \rightarrow 0 .
$$

We shall explain the results related to the main theorem. First it should be noted that the total scattering cross section with incident direction fixed is not necessarily expected to be bounded uniformly in $h, 0<h \ll 1$. It may grow exponentially as $h \rightarrow 0$ for some incident direction in a trapping energy range because of the resonance effect. This result is known as the Breit-Wigner formula in the potential scattering case (for example, see [7]). The uniform boundedeness as $h \rightarrow 0$ of averaged total cross sections has been verified by Sobolev [17] in the potential scattering case. The study there has been made on the bound for $\sigma_{\text {avtot }}(k, g V)$, where $\sigma_{\text {avtot }}(k, g V)$ denotes the averaged total cross section at energy $k$ for Schrödinger operator $-\Delta+g V$ with finite-range potential $V$. The semi-classical result follows as a special case from the bound in the parameter range $(g, k)$ with $k \sim g$ and $g \rightarrow \infty$. On the other hand, the magnetic scattering case corresponds to the parameter range with $k \sim g^{2}$ and $g \rightarrow \infty$ (see (1.13)). The proof of the main theorem is, in principle, based on the same idea as developed in [17]. However the idea there cannot directly apply to the magnetic scattering case. The Hamiltonian $H_{\beta}$ defined by (1.8) has the magnetic string $B(x)=$ $\beta \nabla \gamma(x)$ with strong singularity at the origin. Several new improvements are required at many stages of the proof to control the perturbation $H-H_{\beta}$.

We conclude the section by fixing several basic notations. The notations $\langle$,$\rangle and ($,$) denote the scalar products in R^{2}$ and in $L^{2}\left(R^{2}\right)$, respectively, and \|\| is used to denote the norm of bounded operators. We also fix $\psi \in C_{0}^{\infty}([0, \infty))$ as a basic smooth cut-off function. This function has the following properties: $\psi \geq 0$ is non-increasing and takes the values

$$
\psi(s)=1 \text { for } 0 \leq s \leq 1, \quad \psi(s)=0 \text { for } s>2 .
$$


These notations are often used without further references throughout the entire discussion.

\section{$\S 2$. Integral kernel of operator $T(\lambda ; b)$}

We keep the same notations as in the previous section. In this section, we give the representations for the integral kernel $T(\theta, \omega, \lambda ; b)$ of operator $T(\lambda ; b)$ defined by (1.10) and for the basic quantity

$$
\sigma_{\mathrm{av}}(\lambda ; b)=(2 \pi)^{-1} \int \sigma(\lambda, \omega ; b) d \omega=(2 \pi)^{2}(2 \lambda)^{-1 / 2}\|T(\lambda ; b)\|_{\mathrm{HS}}^{2},
$$

where \|\|$_{\text {HS }}$ denotes the Hilbert-Schmidt norm.

2.1. The representation for the kernel $T(\theta, \omega ; \lambda ; b)$ has been already derived in $[19]$. We begin by making a brief review on the results obtained there. Let $G_{R}=\{x:|x|<R\}$ be the ball centered at the origin with radius $R$. For notational brevity, the magnetic field $b$ is always assumed to be supported in the unit ball

$$
\operatorname{supp} b \subset G_{1} \text {. }
$$

Let $A(x)$ be defined by (1.4) and let $B(x)=\beta \nabla \gamma(x)$ be as in (1.8). Then it readily follows that

$$
\partial_{x}^{\alpha}(A(x)-B(x))=O\left(|x|^{-2-|\alpha|}\right), \quad|x| \rightarrow \infty .
$$

Hence we can define $a(x) \in C^{\infty}\left(R^{2} \backslash\{0\}\right)$ as

$$
a(x)=-\int_{1}^{\infty}\left(x_{1} a_{1}(s x)+x_{2} a_{2}(s x)\right) d s, \quad x \neq 0 .
$$

The function $a(x)$ is not necessarily smooth at the origin. It behaves like $O\left(|x|^{-1}\right)$ as $|x| \rightarrow 0$. A simple computation yields ([19] Lemma 2.2) that

$$
A(x)=B(x)+\nabla a(x)+E(x), \quad x \neq 0,
$$

where $E(x)=\left(e_{1}(x), e_{2}(x)\right)$ is given as

$$
e_{1}(x)=\int_{1}^{\infty} s x_{2} b(s x) d s, \quad e_{2}(x)=-\int_{1}^{\infty} s x_{1} b(s x) d s .
$$

The vector potential $E(x)$ has support in $G_{1}$ but is not necessarily smooth at the origin. 
Let $a(x)$ be as above and let $\psi(s), s \in[0, \infty)$, be a basic smooth cut-off function. It has the properties in (1.14). We often write $\psi_{m}(x)=\psi\left(|x| / 2^{m}\right)$. We now define

$$
V=H J-J H_{\beta}=H(A) J-J H(B),
$$

where $J$ is the multiplication operator with function $J(x)$ defined by

$$
J(x)=\psi_{\infty}(x) J_{a}(x), \quad J_{a}(x)=\exp (i a(x)),
$$

and $\psi_{\infty}(x)=1-\psi_{0}(x), \psi_{0}(x)=\psi(|x|)$. We calculate

$$
V=J_{a}\left(H(A-\nabla a) \psi_{\infty}-\psi_{\infty} H(B)\right)
$$

by use of the gauge transformation. By definition, $\psi_{\infty}(x)$ vanishes on $G_{1}$ and hence it follows from (2.2) that $A-\nabla a=B$ on the support of $\psi_{\infty}$. Since $\psi_{\infty}=\psi_{\infty}(|x|)$ is rotationally invariant, this implies that

$$
V=J_{a}\left[H(B), \psi_{\infty}\right]=J_{a}\left[H_{0}, \psi_{\infty}\right]=J_{a}\left[\psi_{0}, H_{0}\right],
$$

where the notation $[$,$] denotes the commutator relation. Thus the coef-$ ficients of first order differential operator $V$ are all supported in $\{x: 1<$ $|x|<2\}$ and, in particular, they vanish in a neighborhood of the origin.

2.2. As previously stated, the operator $H_{\beta}=H(B)$ admits the partial wave expansion. Let $\Lambda_{l}, l \in Z$, be the eigenspace of the operator $-i \partial / \partial \theta$ acting on $L^{2}\left(S^{1}\right)$ with eigenvalue $l$. Then we have

$$
L^{2}((0, \infty) ; d r) \otimes L^{2}\left(S^{1}\right)=\sum_{l \in Z} \oplus\left(L^{2}((0, \infty) ; d r) \otimes \Lambda_{l}\right) .
$$

We use the unitary mapping

$$
(\mathcal{U} u)(r, \theta)=r^{1 / 2} u(r \theta): L^{2}\left(R^{2}\right) \rightarrow L^{2}((0, \infty) ; d r) \otimes L^{2}\left(S^{1}\right)
$$

to obtain that $H_{\beta}$ is formally decomposed as

$$
H_{\beta} \simeq \mathcal{U} H_{\beta} \mathcal{U}^{-1}=\sum_{l \in Z} \oplus\left(P_{l} \otimes I d\right),
$$

where

$$
P_{l}=\left(-\partial_{r}^{2}+\left(\nu^{2}-1 / 4\right) r^{-2}\right) / 2, \quad \nu=|l-\beta| .
$$


The operator $P_{l}$ has a self-adjoint realization in $L^{2}((0, \infty) ; d r)$ with domain

$$
\mathcal{D}\left(P_{l}\right)=\left\{f \in L^{2}((0, \infty)): P_{l} f \in L^{2}((0, \infty)), \lim _{r \rightarrow 0} r^{-1 / 2}|f(r)|<\infty\right\},
$$

where the operation $P_{l} f$ is interpreted in the distributional sense. Hence the operator $H_{\beta}$ formally defined by (1.8) also admits a self-adjoint realization (denoted by the same symbol $H_{\beta}$ ) in $L^{2}\left(R^{2}\right)$ with domain

$$
\mathcal{D}\left(H_{\beta}\right)=\sum_{l \in Z} \oplus \mathcal{U}^{-1}\left(\mathcal{D}\left(P_{l}\right) \otimes \Lambda_{l}\right)
$$

Let $\gamma(x ; \omega)$ be the azimuth angle from $\omega$. We write

$$
\varphi_{0}(x, \lambda, \omega)=\exp (i \sqrt{2 \lambda}\langle x, \omega\rangle), \quad H_{0} \varphi_{0}=\lambda \varphi_{0}
$$

for the generalized eigenfunction of the free Hamiltonian $H_{0}=-\Delta / 2$. As is well known, $\varphi_{0}$ is expanded as

$$
\varphi_{0}(x, \lambda, \omega)=\sum_{l \in Z} \exp (i|l| \pi / 2) \exp (i l \theta(x)) J_{|l|}(\sqrt{2 \lambda}|x|),
$$

where $\theta(x)=\gamma(x ; \omega)$ and $J_{p}$ denotes the Bessel function of order $p$. We also define the generalized eigenfunctions of operator $H_{\beta}$. These eigenfunctions are formally defined as

$$
\varphi_{ \pm}(x, \lambda, \omega)=W_{ \pm}\left(H_{\beta}, H_{0}\right) \varphi_{0}
$$

by use of the intertwining property. However this definition does not have the rigorous meaning, because $\varphi_{0}$ is not in $L^{2}\left(R^{2}\right)$. To give the precise definition, we make use of the expansion formula (2.7) for $\varphi_{0}$ and of the well-known asymptotic formula of the Bessel functions

$$
J_{p}(r)=(2 / \pi)^{1 / 2} r^{-1 / 2} \cos (r-(2 p+1) \pi / 4)\left(1+u_{N}(r)\right)+O\left(r^{-N}\right)
$$

as $r \rightarrow \infty$, where $u_{N}(r)$ obeys the bound $(d / d r)^{m} u_{N}(r)=O\left(r^{-1-m}\right)$. We now define $f_{ \pm l}(r)$ as

$$
f_{ \pm l}(r)=\exp ( \pm i|l| \pi / 2) J_{|l|}(r)-\exp ( \pm i \nu \pi / 2) J_{\nu}(r), \quad \nu=|l-\beta| .
$$

Then it follows from (2.8) that

$$
f_{ \pm l}(r)=C_{ \pm l} r^{-1 / 2} \exp (\mp i r)+\exp (\mp i r) O\left(r^{-3 / 2}\right)+\exp ( \pm i r) O\left(r^{-3 / 2}\right)
$$


for some constants $C_{ \pm l}$. This relation yields that

$$
(d / d r) f_{ \pm l}(r) \pm i f_{ \pm l}(r)=O\left(r^{-3 / 2}\right), \quad r \rightarrow \infty .
$$

Hence $f_{+l}(r)$ and $f_{-l}(r)$ satisfy the incoming and outgoing radiation conditions at infinity, respectively. Let $\theta(x)=\gamma(x ; \omega)$ be again the azimuth angle from $\omega$ and let $\zeta(x)=\gamma(x ;-\omega)$. If we make use of expansion formula $(2.7)$ and of the simple relation $\exp (i|l| \pi+i l \theta)=\exp (i l \zeta)$, then we can define the incoming eigenfunction $\varphi_{+}$associated with wave operator $W_{+}\left(H_{\beta}, H_{0}\right)$ as

$$
\varphi_{+}(x, \lambda, \omega)=\sum_{l \in Z} \exp (i \nu \pi / 2) \exp (i l \theta(x)) J_{\nu}(\sqrt{2 \lambda}|x|), \quad \nu=|l-\beta|
$$

and the outgoing eigenfunction $\varphi_{-}$associated with $W_{-}\left(H_{\beta}, H_{0}\right)$ as

$$
\varphi_{-}(x, \lambda, \omega)=\sum_{l \in Z} \exp (-i \nu \pi / 2) \exp (i l \zeta(x)) J_{\nu}(\sqrt{2 \lambda}|x|), \nu=|l-\beta|
$$

As is easily seen, the two eigenfunctions above are connected with each other through the relation

$$
\varphi_{+}(x, \lambda, \omega)=\exp (i(\nu-l) \pi) \varphi_{-}(x, \lambda, \omega) .
$$

The scattering matrix $S\left(\lambda ; H_{\beta}, H_{0}\right)$ has the property

$$
S\left(\lambda ; H_{\beta}, H_{0}\right): \bar{\varphi}_{-}(x, \lambda, \cdot) \rightarrow \bar{\varphi}_{+}(x, \lambda, \cdot)
$$

as an operator acting on $L^{2}\left(S^{1}\right)=\sum_{l \in Z} \oplus \Lambda_{l}$. This implies that $S(\lambda$; $\left.H_{\beta}, H_{0}\right)$ acts as the multiplication operator with $\exp (i(l-\nu) \pi)$ on each eigenspace $\Lambda_{l}$ and hence the integral kernel of $S\left(\lambda ; H_{\beta}, H_{0}\right)$ is given by

$$
S\left(\theta, \omega ; \lambda ; H_{\beta}, H_{0}\right)=(2 \pi)^{-1} \sum_{l=-\infty}^{\infty} \exp (i(l-\nu) \pi) \exp (i l(\theta-\omega)),
$$

which leads us to (1.9) after a simple calculation. Here the coordinates over $S^{1}$ are identified with the azimuth angles from the positive $x_{1}$ axis.

2.3. We write $R(\mu ; H), \operatorname{Im} \mu \neq 0$, for the resolvent $(H-\mu)^{-1}$. Let

$$
L_{s}^{2}\left(R^{2}\right)=L^{2}\left(R^{2} ;\langle x\rangle^{2 s} d x\right), \quad\langle x\rangle=\left(1+|x|^{2}\right)^{1 / 2}
$$


be a weighted $L^{2}$ space. According to the results due to [9], the operator $H$ has the following spectral properties: (1) $H$ has no bound states; (2) The boundary values $R(\lambda \pm i 0 ; H), \lambda>0$, of resolvents to the positive real axis exist

$$
R(\lambda \pm i 0 ; H)=\lim _{\epsilon \downarrow 0} R(\lambda \pm i \epsilon ; H): L_{s}^{2}\left(R^{2}\right) \rightarrow L_{-s}^{2}\left(R^{2}\right)
$$

for $s>1 / 2$, where the convergence is locally uniform in $\lambda>0$. The lemma below has been obtained as Proposition 5.1 of [19].

LEMmA 2.1. Let $V$ and $J$ be defined by (2.3) and (2.4), respectively. Then the operator $T(\lambda ; b)$ has the following integral kernel:

$$
T(\theta, \omega, \lambda ; b)=(2 \pi)^{-2}\left(\left(J^{*}-V^{*} R(\lambda+i 0 ; H)\right) V \varphi_{-}(\lambda, \omega), \varphi_{+}(\lambda, \theta)\right),
$$

where ( , ) denotes the $L^{2}$ scalar product in $L^{2}\left(R^{2}\right)$ and $\varphi_{ \pm}(\lambda, \omega)=$ $\varphi_{ \pm}(x, \lambda, \omega)$ are the eigenfunctions of $H_{\beta}$ defined by (2.9) and (2.10).

This lemma shows that $T(\lambda ; b): L^{2}\left(S^{1}\right) \rightarrow L^{2}\left(S^{1}\right)$ is of Hilbert-Schmidt class for all $\lambda>0$ and hence it follows by definition that

$$
\sigma_{\mathrm{av}}(\lambda ; b)=(2 \pi)^{2}(2 \lambda)^{-1 / 2}\left\|\left(T^{*} T\right)(\lambda ; b)\right\|_{\mathrm{Tr}},
$$

where $\left(T^{*} T\right)(\lambda ; b)=T^{*}(\lambda ; b) T(\lambda ; b)$ and \|\|$_{T r}$ stands for the trace norm. We calculate the integral kernel of $\left(T^{*} T\right)(\lambda ; b)$ by making use of Lemma 2.1. For notational brevity, we write $S(\lambda)$ for $S\left(\lambda ; H, H_{0}\right)$ and $S_{\beta}(\lambda)$ for $S\left(\lambda ; H_{\beta}, H_{0}\right)$. Since both the operators are unitary, we obtain

$$
\left(T^{*} T\right)(\lambda ; b)=i(2 \pi)^{-1}\left\{S_{\beta}^{*}(\lambda) T(\lambda ; b)-\left(S_{\beta}^{*}(\lambda) T(\lambda ; b)\right)^{*}\right\} .
$$

By $(2.11), S_{\beta}^{*}(\lambda)$ maps $\bar{\varphi}_{+}(x, \lambda, \cdot)$ to $\bar{\varphi}_{-}(x, \lambda, \cdot)$ and hence the integral kernel of $\left(S_{\beta}^{*} T\right)(\lambda ; b)$ is represented as

$$
\left(S_{\beta}^{*} T\right)(\theta, \omega ; \lambda ; b)=(2 \pi)^{-2}\left(\left(J^{*}-V^{*} R(\lambda+i 0 ; H(A))\right) V \varphi_{-}(\lambda, \omega), \varphi_{-}(\lambda, \theta)\right)
$$

by Lemma 2.1. Thus the kernel of operator $\left(T^{*} T\right)(\lambda ; b)$ is decomposed into the sum $T_{1}(\theta, \omega, \lambda ; b)+T_{2}(\theta, \omega, \lambda ; b)$, where

$$
\begin{aligned}
& T_{1}=i(2 \pi)^{-3}\left(\left(J^{*} V-V^{*} J\right) \varphi_{-}(\lambda, \omega), \varphi_{-}(\lambda, \theta)\right), \\
& T_{2}=(2 \pi)^{-2}\left(E^{\prime}(\lambda ; H) V \varphi_{-}(\lambda, \omega), V \varphi_{-}(\lambda, \theta)\right)
\end{aligned}
$$


and $E^{\prime}(\lambda ; H)$ is given as

$$
E^{\prime}(\lambda ; H)=(d / d \lambda) E(\lambda ; H)=(2 \pi i)^{-1}(R(\lambda+i 0 ; H)-R(\lambda-i 0 ; H))
$$

with the spectral resolution $E(\lambda ; H)$ of $H$. We assert that $T_{1}(\theta, \omega, \lambda ; b)=0$. To see this, we recall the definition (2.3) of $V$ and compute

$$
J^{*} V-V^{*} J=\left[1-\psi_{\infty}^{2}, H_{\beta}\right]
$$

where $1-\psi_{\infty}^{2}$ has compact support. Since $H_{\beta} \varphi_{-}=\lambda \varphi_{-}$, the above assertion follows at once. Thus the representation for the integral kernel of $\left(T^{*} T\right)(\lambda ; b)$ is obtained as

$$
\left(T^{*} T\right)(\theta, \omega, \lambda ; b)=(2 \pi)^{-2}\left(E^{\prime}(\lambda ; H) V \varphi_{-}(\lambda, \omega), V \varphi_{-}(\lambda, \theta)\right) .
$$

Recall that all the coefficients of $V$ are supported in $\{x: 1<|x|<2\}$. Let $\psi_{2}(x)=\psi\left(|x| / 2^{2}\right)$, so that $\psi_{2} V=V \psi_{2}=V$. Taking account of these facts, we now define the following two operators:

$$
\begin{aligned}
(Z(\lambda) f)(x) & =\int\left(V \varphi_{-}\right)(x, \lambda, \omega) f(\omega) d \omega: L^{2}\left(S^{1}\right) \rightarrow L^{2}\left(R^{2}\right), \\
\Gamma(\lambda) & =\psi_{2} E^{\prime}(\lambda ; H) \psi_{2}: L^{2}\left(R^{2}\right) \rightarrow L^{2}\left(R^{2}\right) .
\end{aligned}
$$

Then we combine (2.12) and (2.13) to obtain the following

LEMma 2.2. Let the notations be as above. Then the operator $\left(T^{*} T\right)$ $(\lambda ; b)$ is decomposed as the composition of three operators

$$
\left(T^{*} T\right)(\lambda ; b)=(2 \pi)^{-2} Z^{*}(\lambda) \Gamma(\lambda) Z(\lambda)
$$

and one has

$$
\sigma_{\mathrm{av}}(\lambda ; b)=(2 \lambda)^{-1 / 2}\left\|Z^{*}(\lambda) \Gamma(\lambda) Z(\lambda)\right\|_{\operatorname{Tr}}
$$

2.4. We turn back to the semi-classical case. We use $g$ and $k$ with the meanings ascribed in (1.13). Taking account of relation (1.12), we define the two Hamiltonians as follows:

$$
H(g)=H(g A)=(-i \nabla-g A)^{2} / 2, \quad H_{\beta}(g)=H(g B)=(-i \nabla-g B)^{2} / 2 .
$$

The outgoing eigenfunction $\varphi_{-}(x, k, \omega ; g)$ of $H_{\beta}(g)$ is given by

$$
\varphi_{-}=\sum_{l \in Z} \exp (-i \nu \pi / 2) \exp (i l \zeta(x)) J_{\nu}(\sqrt{2 k}|x|), \quad \nu=|l-g \beta|,
$$


with the azimuth angle $\zeta(x)=\gamma(x ;-\omega)$ from $-\omega$. We further define the following operators:

$$
\begin{aligned}
& J(g)=\psi_{\infty}(x) \exp (i g a(x)) \times, \quad J_{a}(g)=\exp (i g a(x)) \times, \\
& V(g)=H(g) J(g)-J(g) H_{\beta}(g)=J_{a}(g)\left[\psi_{0}, H_{0}\right], \\
& (Z(k ; g) f)(x)=\int\left(V(g) \varphi_{-}\right)(x, k, \omega ; g) f(\omega) d \omega: L^{2}\left(S^{1}\right) \rightarrow L^{2}\left(R^{2}\right), \\
& \Gamma(k ; g)=\psi_{2} E^{\prime}(k ; H(g)) \psi_{2}: L^{2}\left(R^{2}\right) \rightarrow L^{2}\left(R^{2}\right),
\end{aligned}
$$

where $a(x)$ is defined by $(2.1)$ and

$$
E^{\prime}(k ; H(g))=(2 \pi i)^{-1}\{R(k+i 0 ; H(g))-R(k-i 0 ; H(g))\} .
$$

Then it follows from Lemma 2.2 that the quantity $\sigma_{\mathrm{av}, h}(\lambda ; b)$ in Theorem 1.1 is expressed as

$$
\sigma_{\mathrm{av}, h}(\lambda ; b)=\sigma_{\mathrm{av}}(k ; g b)=(2 k)^{-1 / 2}\left\|Z^{*}(k ; g) \Gamma(k ; g) Z(k ; g)\right\|_{\operatorname{Tr}}
$$

with $g=h^{-1}$ and $k=\lambda h^{-2}=\lambda g^{2}$. This relation plays a basic role in proving the main theorem.

\section{$\S 3 . \quad$ Main lemmas and proof of Theorem 1.1}

In this section we prove Theorem 1.1, accepting the two main lemmas below (Lemmas 3.2 and 3.3) as proved. The main body of the present work is occupied by the proof of these two lemmas.

3.1. The proof of the theorem is based on the singular number $(s$ number) theory of bounded operators. Let $X_{j}, 1 \leq j \leq 2$, be separable Hilbert spaces and let $B_{n}=B_{n}\left(X_{1}, X_{2}\right)$ denote the class of finitedimensional operators $K: X_{1} \rightarrow X_{2}$ with $\operatorname{dim} \operatorname{Ran} K \leq n$. For a bounded operator $F: X_{1} \rightarrow X_{2}$, we define the singular numbers of $F$ as follows:

$$
s_{n+1}(F)=\min _{K \in B_{n}}\|F-K\|, \quad n \geq 0,
$$

where \|\| stands for the norm of operators from $X_{1}$ into $X_{2}$. If, in particular, $F: X_{1} \rightarrow X_{1}$ is a non-negative compact operator, then the $s$-numbers $s_{n}(F)$ are nothing but the eigenvalues of $F$. We here summarize several basic properties of $s$-numbers which are repeatedly used throughout the argument below. We refer to [8] and [18] (Lemma 3) for the proof of these properties. 
Proposition 3.1. (1) $s_{n}(F)=s_{n}\left(F^{*}\right)$.

(2) Let $F_{1}, F_{2}: X_{1} \rightarrow X_{2}$ be bounded operators. Then

$$
s_{n+m-1}\left(F_{1}+F_{2}\right) \leq s_{n}\left(F_{1}\right)+s_{m}\left(F_{2}\right)
$$

(3) Let $F_{1}: X_{1} \rightarrow X_{2}$ and $F_{2}: X_{2} \rightarrow X_{3}$ be bounded operators. Then

$$
s_{n+m-1}\left(F_{2} F_{1}\right) \leq s_{n}\left(F_{1}\right) s_{m}\left(F_{2}\right) .
$$

(4) Let $F: X_{1} \rightarrow X_{1}$ be a compact operator. Suppose that $(F+I d)^{-1}$ : $X_{1} \rightarrow X_{1}$ is also bounded. If $s_{n}(F)<1$, then

$$
s_{n}\left((F+I d)^{-1}\right) \leq\left(1-s_{n}(F)\right)^{-1} .
$$

We now formulate the two main lemmas from which Theorem 1.1 is obtained by repeated use of Proposition 3.1.

Lemma 3.2. Let $Z(k ; g): L^{2}\left(S^{1}\right) \rightarrow L^{2}\left(R^{2}\right)$ be defined in $(2.15)$. Then there exists $L \gg 1$ large enough such that for $n>L g$

$$
s_{n}(Z(k ; g)) \leq C e^{-d n}
$$

with some $C, d>0$ independent of $n$ and $g \gg 1$.

Lemma 3.3. Let $\Gamma(k ; g): L^{2}\left(R^{2}\right) \rightarrow L^{2}\left(R^{2}\right)$ be defined in $(2.15)$. Then there exists $M \gg 1$ such that for $n>M g$

$$
s_{n}(\Gamma(k ; g)) \leq C g^{3}
$$

with some $C>0$ independent of $n$ and $g \gg 1$.

3.2. We first complete the proof of Theorem 1.1, accepting the two lemmas above as proved.

Proof of Theorem 1.1. We write

$$
e_{n}=e_{n}(k ; g)=s_{n}\left(Z(k ; g)^{*} \Gamma(k ; g) Z(k ; g)\right), \quad n \geq 1
$$


Then it follows from (2.16) that

$$
\sigma_{\mathrm{av}}(k ; g b)=(2 k)^{-1 / 2} \sum_{n=1}^{\infty} e_{n} .
$$

Let $L$ and $M$ be as in Lemmas 3.2 and 3.3, respectively. We set $J=[L g]$, $N=[M g]$ and $m=N+2 J$. Then the sum above is decomposed into

$$
\sum_{n=1}^{\infty} e_{n}=I_{0}(k ; g)+I_{1}(k ; g)+I_{2}(k ; g)
$$

where

$$
I_{0}=\sum_{n=1}^{m} e_{n}, \quad I_{1}=\sum_{j=1}^{\infty} e_{m+2 j-1}, \quad I_{2}=\sum_{j=1}^{\infty} e_{m+2 j} .
$$

Note that $k=\lambda g^{2}, \lambda>0$ being fixed. Since $e_{n} \leq 4$ for all $n \geq 1$ by Lemma 2.2 , we have

$$
(2 k)^{-1 / 2} I_{0}(k ; g)=O(1), \quad g \rightarrow \infty .
$$

We evaluate the other terms. We decompose

$$
m+2 j-1=(N+1)+2(J+j)-2
$$

and use Proposition 3.1 to obtain that

$$
I_{1}(k ; g) \leq s_{N+1}(\Gamma(k ; g)) \sum_{j=1}^{\infty} s_{J+\jmath}(Z(k ; g))^{2} .
$$

This, together with Lemmas 3.2 and 3.3, yields the bound

$$
(2 k)^{-1 / 2} I_{1}(k ; g)=O(1) .
$$

Similarly we can show that $I_{2}(k ; g)$ also obeys the same bound as above. Thus the proof is complete.

\section{$\S 4$. Proof of Lemma 3.2}

We first prove Lemma 3.2. This lemma is easy to prove. We begin by recalling the definition $(2.14)$ of eigenfunction $\varphi$ -

$$
\varphi_{-}(x, k, \omega ; g)=\sum_{l \in Z} \exp (-i \nu \pi / 2) \exp (i l \zeta(x)) J_{\nu}(\sqrt{2 k}|x|), \quad \nu=|l-g \beta|,
$$

where $\zeta(x)=\gamma(x ;-\omega)$ is again the azimuth angle from $-\omega$. 
Lemma 4.1. Assume that $r$ is in a compact interval $[0, R]$. Then there exists $L \gg 1$ such that for $\nu>L g$

$$
\left|J_{\nu}(\sqrt{2 k} r)\right|+\left|(d / d r) J_{\nu}(\sqrt{2 k} r)\right| \leq C e^{-d \nu}
$$

with some d, $C>0$ independent of $\nu$.

Proof. The proof uses the Poisson integral representation for the Bessel function ([5] p.81)

$$
J_{p}(r)=(r / 2)^{p} \frac{1}{\sqrt{\pi} \Gamma(p+1 / 2)} \int_{0}^{\pi} \cos (r \cos \theta) \sin ^{2 p} \theta d \theta
$$

and the Stirling formula for the gamma function

$$
\Gamma(p)=(2 \pi)^{1 / 2} e^{-p} p^{p-1 / 2}(1+o(1)), \quad p \rightarrow \infty .
$$

If $\nu>L g$ for $L \gg 1$, then it follows from these two relations that

$$
\left|J_{\nu}(\sqrt{2 k} r)\right| \leq C\left(c k / \nu^{2}\right)^{\nu / 2} \leq C(1 / 2)^{\nu}, \quad r \in[0, R],
$$

for some $c, C>0$. A similar argument applies to $(d / d r) J_{\nu}(\sqrt{2 k} r)$ for $\nu \geq L g$ with another $L \gg 1$. This completes the proof.

Proof of Lemma 3.2. A simple consideration yields

$$
\exp (i l \zeta(x))=\exp (i l \gamma(x ;-\omega))=\exp (i l \gamma(x)) \exp (i l(\pi-\omega))
$$

where $\gamma(x)$ is again the azimuth angle from the positive $x_{1}$ axis. Recall the definition (2.15) of operator $V(g), V(g)=J_{a}(g)\left[\psi_{0}, H_{0}\right]$, where $\psi_{0}=$ $\psi(|x|)$ is a function of $|x|$ only and $J_{a}(g)$ is the multiplication operator with $\exp (i g a(x)), a(x)$ being defined by (2.1). Hence we see from Lemma 4.1 that the integral kernel of operator $Z(k ; g): L^{2}\left(S^{1}\right) \rightarrow L^{2}\left(R^{2}\right)$ is represented in the form

$$
Z(x, \omega ; k, g)=\sum_{l \in Z} f_{\nu}(x ; k, g) \otimes \exp (-i l \omega), \quad \nu=|l-g \beta|
$$

where $f_{\nu}$ has support in $\{x: 1<|x|<2\}$ for all $\nu$ and obeys the estimate

$$
\left|f_{\nu}(x ; k, g)\right| \leq C e^{-d \nu}, \quad \nu>L g
$$


with the same constants $d, L$ as in Lemma 4.1. Assume that $n>L_{1} g$ for some $L_{1} \gg 1$. We choose $L_{1}$ so large that

$$
|l| \geq n / 2>L_{1} g / 2 \Longrightarrow \nu=|l-\beta g|>n / 3>L g
$$

and we denote by $Z_{n}(k ; g): L^{2}\left(S^{1}\right) \rightarrow L^{2}\left(R^{2}\right)$ the integral operator with kernel

$$
Z_{n}(x, \omega ; k, g)=\sum_{|l|<[n / 2]} f_{\nu}(x ; k, g) \otimes \exp (-i l \omega)
$$

for $n$ as above. As is easily seen, $\operatorname{dim} \operatorname{Ran} Z_{n}(k ; g) \leq n-1$, so that

$$
s_{n}(Z(k ; g)) \leq\left\|Z(k ; g)-Z_{n}(k ; g)\right\| \leq C e^{-d n}
$$

for some $d, C>0$, which follows from (4.3) at once. This proves the lemma.

\section{$\S 5$. Proof of Lemma 3.3}

The proof of Lemma 3.3 is rather long. The present and following three sections are devoted to the proof of this lemma. The proof is based on the three key lemmas (Lemmas $5.1 \sim 5.3$ ) formulated below. We here complete the proof of Lemma 3.3, accepting these three lemmas as proved.

5.1. We first derive several basic relations which are required to formulate the key lemmas above. We decompose the magnetic potential $A=A(x)$ into $A=\psi_{\infty} A+\psi_{0} A$. We further rewrite $A$ by use of relation (2.2). Let $E(x)$ be as in (2.2). Since $\psi_{\infty} E=0, A$ is written as

$$
A=\psi_{\infty} B+a \nabla \psi_{0}+\psi_{0} A+\nabla\left(\psi_{\infty} a\right)=A_{1}+\nabla\left(\psi_{\infty} a\right) .
$$

We set $B_{1}=a \nabla \psi_{0}+\psi_{0} A$, so that $A_{1}=\psi_{\infty} B+B_{1}$. As is easily seen, $A_{1}(x)$ and $B_{1}(x)$ are smooth functions and, in particular, $B_{1}(x)$ has support in $G_{2}=\{x:|x|<2\}$.

We here introduce the following two Hamiltonians:

$$
\begin{aligned}
& K(g)=\left(-i \nabla-g A_{1}\right)^{2} / 2, \\
& K_{\beta}(g)=\left(-i \nabla-g \psi_{\infty} B\right)^{2} / 2+N g^{2} \psi_{1}
\end{aligned}
$$

with $N \gg 1$, where $\psi_{1}=\psi(|x| / 2)$ has support in $G_{4}$. The choice of $N$ is specified in the later discussion (see Lemma 6.1). Obviously both the Hamiltonians admit self-adjoint realizations in $L^{2}\left(R^{2}\right)$ with the same domains $\mathcal{D}(K(g))=\mathcal{D}\left(K_{\beta}(g)\right)=H^{2}\left(R^{2}\right)$. The Hamiltonian $K(g)$ is unitarily 
equivalent to the orginal one $H(g)$, which follows from (5.1) at once, and also $K_{\beta}(g)$ has the partial wave expansion. Let

$$
W(g)=K(g)-K_{\beta}(g)
$$

be the difference between the two Hamiltonians defined above. All the coefficients of first order differential operator $W(g)$ have support in $G_{4}$ and obey the bound $O\left(g^{2}\right)$ as $g \rightarrow \infty$. Hence it satisfies the relation

$$
\psi_{2} W(g)=W(g) \psi_{2}=W(g),
$$

because $\psi_{2}=\psi\left(|x| / 2^{2}\right)=1$ on $G_{4}$. Taking account of this fact, we obtain from the resolvent equation that

$$
\begin{aligned}
& \psi_{2} W(g)\left\{R(\mu ; K(g))-R\left(\mu ; K_{\beta}(g)\right)\right\} \psi_{2} \\
& \quad=-\psi_{2} W(g) R(\mu ; K(g)) \psi_{2} \cdot \psi_{2} W(g) R\left(\mu ; K_{\beta}(g)\right) \psi_{2}
\end{aligned}
$$

with $\mu=k+i \epsilon, 0<\epsilon \ll 1$, and hence we have

$$
\left(I d-\psi_{2} W(g) R(\mu ; K(g)) \psi_{2}\right)\left(I d+\psi_{2} W(g) R\left(\mu ; K_{\beta}(g)\right) \psi_{2}\right)=I d .
$$

Similarly we have

$$
\left(I d+\psi_{2} W(g) R\left(\mu ; K_{\beta}(g)\right) \psi_{2}\right)\left(I d-\psi_{2} W(g) R(\mu ; K(g)) \psi_{2}\right)=I d,
$$

so that

$$
\left(I d+\psi_{2} W(g) R\left(\mu ; K_{\beta}(g)\right) \psi_{2}\right)^{-1}=\left(I d-\psi_{2} W(g) R(\mu ; K(g)) \psi_{2}\right)
$$

It follows again from the resolvent equation that

$$
\psi_{2} R(\mu ; K(g)) \psi_{2}\left(I d+\psi_{2} W(g) R\left(\mu ; K_{\beta}(g)\right) \psi_{2}\right)=\psi_{2} R\left(\mu ; K_{\beta}(g)\right) \psi_{2},
$$

which implies that

(5.5) $\psi_{2} R(\mu ; K(g)) \psi_{2}=\psi_{2} R\left(\mu ; K_{\beta}(g)\right) \psi_{2}\left(I d+\psi_{2} W(g) R\left(\mu ; K_{\beta}(g)\right) \psi_{2}\right)^{-1}$.

5.2. We denote by $K^{D}(g)$ the self-adjoint operator obtained from $K(g)$ by imposing the zero Dirichlet conditions on the boundary $\partial G_{9}$ of $G_{9}=\{x:|x|<9\}$. In other words, $K^{D}(g)=K(g)$ as a differential operator and its domain is

$$
\mathcal{D}\left(K^{D}(g)\right)=\left(H_{0}^{1}\left(G_{9}\right) \cap H^{2}\left(G_{9}\right)\right) \oplus\left(H_{0}^{1}\left(G_{9}^{c}\right) \cap H^{2}\left(G_{9}^{c}\right)\right) .
$$


We also define $K_{\beta}^{D}(g)$ for $K_{\beta}(g)$ in a similar way. Both the operators are regarded as an operator acting on $L^{2}\left(R^{2}\right)$ and their difference is still

$$
W(g)=K^{D}(g)-K_{\beta}^{D}(g)=K(g)-K_{\beta}(g) .
$$

The support of $\psi_{2}$ is completely contained in $G_{9}$; supp $\psi_{2} \subset G_{8} \subset G_{9}$. Thus we repeat the same argument as above to obtain

$$
\left(I d+\psi_{2} W(g) R\left(\mu ; K_{\beta}^{D}(g)\right) \psi_{2}\right)^{-1}=\left(I d-\psi_{2} W(g) R\left(\mu ; K^{D}(g)\right) \psi_{2}\right) .
$$

We now decompose the operator $\psi_{2} R(\mu ; K(g)) \psi_{2}$ in (5.5) into the composition of three operators

$$
\psi_{2} R(\mu ; K(g)) \psi_{2}=F_{1}(\mu ; g) F_{2}(\mu ; g) F_{3}(\mu ; g)
$$

where

$$
\begin{aligned}
& F_{1}(\mu ; g)=\psi_{2} R\left(\mu ; K_{\beta}(g)\right) \psi_{2}, \\
& F_{2}(\mu ; g)=I d-\psi_{2} W(g) R\left(\mu ; K^{D}(g)\right) \psi_{2}, \\
& F_{3}(\mu ; g)=(I d+Y(\mu ; g))^{-1}
\end{aligned}
$$

with

$$
I d+Y(\mu ; g)=\left(I d+\psi_{2} W(g) R\left(\mu ; K_{\beta}(g)\right) \psi_{2}\right) F_{2}(\mu ; g) .
$$

We can rewrite $Y(\mu ; g)$ as

$$
Y(\mu ; g)=\psi_{2} W(g)\left\{R\left(\mu ; K_{\beta}(g)\right)-R\left(\mu ; K_{\beta}^{D}(g)\right)\right\} \psi_{2} F_{2}(\mu ; g)
$$

by use of (5.6). The second main lemma follows from the three lemmas below. These lemmas are proved in Sections 6, 7 and 8 .

LEMMA 5.1.

$$
\limsup _{\epsilon \rightarrow 0} s_{1}\left(F_{1}(k+i \epsilon ; g)\right) \leq C g^{-1}
$$

with some $C>0$ independent of $g \gg 1$.

LEMma 5.2. There exists $M \gg 1$ such that for $n>M g$

$$
\limsup _{\epsilon \rightarrow 0} s_{n}\left(F_{2}(k+i \epsilon ; g)\right) \leq C g^{4}
$$

with some $C>0$ independent of $g \gg 1$. 
LEMmA 5.3. There exists another $M \gg 1$ such that for $n>M g$

$$
\limsup _{\epsilon \rightarrow 0} s_{n}\left(F_{3}(k+i \epsilon ; g)\right) \leq 2, \quad g \gg 1 .
$$

5.3. We shall complete the proof of Lemma 3.3, accepting the three key lemmas above as proved.

Proof of Lemma 3.3. By the principle of limiting absorption,

$$
\psi_{2} R(k+i \epsilon ; K(g)) \psi_{2} \rightarrow \psi_{2} R(k+i 0 ; K(g)) \psi_{2}, \quad \epsilon \rightarrow 0,
$$

in norm as an bounded operator acting on $L^{2}\left(R^{2}\right)$ and hence we have

$$
s_{n}\left(\psi_{2} R(k+i \epsilon ; K(g)) \psi_{2}\right) \rightarrow s_{n}\left(\psi_{2} R(k+i 0 ; K(g)) \psi_{2}\right), \quad \epsilon \rightarrow 0,
$$

for each $n \geq 1$. By Proposition 3.1 and by the three lemmas above, it follows from (5.7) that

$$
s_{n}\left(\psi_{2} R(k \pm i 0 ; K(g)) \psi_{2}\right) \leq C g^{3}
$$

for $n>M g$ with some $M \gg 1$. Since the two operators $H(g)$ and $K(g)$ are unitarily equivalent to each other as previously stated, this proves the lemma.

\section{Proof of Lemma 5.1}

The present section is devoted to the proof of Lemma 5.1. The proof is reduced to the semi-classical resolvent estimate in a non-trapping energy range.

6.1. We first determine how large $N$ is chosen in (5.2). The Hamiltonian $K_{\beta}(g)$ has

$$
\psi_{\infty} B=\beta \psi_{\infty} \nabla \gamma=\beta \psi_{\infty}(r)\left(-x_{2} / r^{2}, x_{1} / r^{2}\right), \quad r=|x|,
$$

as a magnetic potential. We compute the magnetic field

$$
d \psi_{\infty} B=\beta\left(\psi_{\infty}^{\prime}(r) / r\right) d x_{1} \wedge d x_{2}
$$

Hence the classical system associated with Hamiltonian $K_{\beta}(g)$ is given by

$$
\begin{aligned}
& x_{1}^{\prime \prime}=\beta \psi_{\infty}^{\prime}(|x|) x_{2}^{\prime} /|x|-N \psi_{1}^{\prime}(|x|) x_{1} /|x|, \\
& x_{2}^{\prime \prime}=-\beta \psi_{\infty}^{\prime}(|x|) x_{1}^{\prime} /|x|-N \psi_{1}^{\prime}(|x|) x_{2} /|x| .
\end{aligned}
$$


This classical system conserves the energy

$$
\left(x_{1}^{\prime}(t)^{2}+x_{2}^{\prime}(t)^{2}\right) / 2+N \psi_{1}(x(t))=\text { const. }
$$

We say that energy $\lambda>0$ is in a non-trapping energy range, if all the solutions $x(t ; \lambda)$ with energy $\lambda$ of equation (6.1) escape to infinity, $|x(t ; \lambda)| \rightarrow \infty$, as $t \rightarrow \pm \infty$.

LEMmA 6.1. One can take $N \gg 1$ so large that the energy $\lambda=k^{-2}$ under consideration is in a non-trapping energy range for the classical system (6.1).

Proof. The system (6.1) is spherically symmetric. We consider this in the polar coordinate system $(r, \theta)$. We have the conservation law for two quantities (energy and angular momentum)

$$
\left(\left(r^{\prime}\right)^{2}+r^{2}\left(\theta^{\prime}\right)^{2}\right) / 2+N \psi_{1}(r)=\lambda, \quad r^{2} \theta^{\prime}+\beta \psi_{\infty}(r)=\rho .
$$

Hence it follows that

$$
\left(r^{\prime}\right)^{2} / 2+\left(\rho-\beta \psi_{\infty}(r)\right)^{2} r^{-2} / 2+N \psi_{1}(r)=\lambda
$$

We now choose $N>\lambda$ large enough. Then $N \psi_{1}(r)=N>\lambda$ for $0<r \leq 2$. This implies that $G_{2}$ is a classically forbidden region. On the other hand, if $r>2$, then $\psi_{\infty}(r)=1$ and hence

$$
(d / d r)\left((\rho-\beta)^{2} r^{-2} / 2+N \psi_{1}(r)\right)<0,
$$

because $\psi_{1}^{\prime}=\psi^{\prime}(r / 2) / 2 \leq 0$ (recall that the basic cut-off function $\psi$ is non-increasing). This proves the lemma.

We now fix $N$ as specified in Lemma 6.1. Then Lemma 5.1 is obtained as an immediate consequence of the lemma below.

Lemma 6.2. Let $N$ be as above. Then one has

$$
\langle x\rangle^{-s} R\left(k+i \epsilon ; K_{\beta}(g)\right)\langle x\rangle^{-s}: L^{2}\left(R^{2}\right) \rightarrow L^{2}\left(R^{2}\right), \quad s>1 / 2,
$$

is bounded with bound $O\left(g^{-1}\right)$ as $g \rightarrow \infty$ uniformly in $\epsilon, 0<\epsilon \ll 1$.

Proof. By Lemma 6.1, $\lambda$ is in a non-trapping energy range. Hence the lemma follows from the semi-classical resolvent estimate due to $[6,14]$. 
6.2. For later references, we here prepare two simple lemmas which will be used to prove Lemma 5.3. As previously stated, $K_{\beta}(g)$ admits the partial wave expansion. If we use the unitary mapping $\mathcal{U}$ in (2.6), then this operator is expanded as

$$
K_{\beta}(g) \simeq \sum_{l \in Z} \oplus\left(P_{\beta l}(g) \otimes I d\right)
$$

where

$$
P_{\beta l}(g)=\left(-\partial_{r}^{2}+\left(\left(l-g \beta \psi_{\infty}(r)\right)^{2}-1 / 4\right) r^{-2}\right) / 2+N g^{2} \psi_{1}(r) .
$$

Similarly we have the expansion

$$
K_{\beta}^{D}(g) \simeq \sum_{l \in Z} \oplus\left(P_{\beta l}^{D}(g) \otimes I d\right)
$$

for operator $K_{\beta}^{D}(g)$, where $P_{\beta l}^{D}(g)$ is obtained from $P_{\beta l}(g)$ by imposing the zero Dirichlet boundary condition at $r=9$.

LEMMA 6.3. Let $\chi_{9}=\chi_{9}(r)$ be the characteristic function of the interval $[0,9]$. Then one has that

$$
\chi_{9} R\left(k+i \epsilon ; P_{\beta l}(g)\right) \chi_{9}: L^{2}((0, \infty) ; d r) \rightarrow L^{2}((0, \infty) ; d r)
$$

is bounded with bound $O\left(g^{-1}\right)$ uniformly in $l \in Z$ and $\epsilon$. If, in particular, $|l|>L g$ with some $L \gg 1$, then the operator obeys the bound $O\left(l^{-2}\right)$.

Proof. The first statement is an immediate consequence of Lemma 6.2. The second one readily follows from tha fact that

$$
\left(\left(l-g \beta \psi_{\infty}(r)\right)^{2}-1 / 4\right) r^{-2} \geq C l^{2} \gg k, \quad|l|>L g,
$$

for $0<r<9$, provided that $L \gg 1$.

LEMma 6.4. One can take another $L \gg 1$ so large that for $|l|>L g$

$$
\chi_{9} R\left(k+i \epsilon ; P_{\beta l}^{D}(g)\right) \chi_{9}: L^{2}((0, \infty) ; d r) \rightarrow L^{2}((0, \infty) ; d r)
$$

is bounded with bound $O\left(l^{-2}\right)$ uniformly in $\epsilon$. In other words, $k$ is not the eigenvalue of $P_{\beta l}^{D}(g)$ for $l$ as above and there exists the limit

$$
\chi_{9} R\left(k+i 0 ; P_{\beta l}^{D}(g)\right) \chi_{9}=\lim _{\epsilon \rightarrow 0} \chi_{9} R\left(k+i \epsilon ; P_{\beta l}^{D}(g)\right) \chi_{9}
$$

uniformly in $l$ as a bounded operator on $L^{2}((0, \infty) ; d r)$.

Proof. This lemma also follows from (6.2) at once. 


\section{$\S 7 . \quad$ Proof of Lemma 5.2}

In this section we prove Lemma 5.2. The proof is based on the asymptotic distribution of eigenvalues with sharp remainder estimate.

7.1. Recall the notation $K^{D}(g)$ in (5.2). It has

$$
K(x, \xi ; g)=\left|\xi-g A_{1}(x)\right|^{2} / 2, \quad(x, \xi) \in R^{2 \times 2}
$$

as its symbol. We denote by $K_{\text {in }}^{D}(g)$ the part of the operator $K^{D}(g)$ restricted to the interior domain $G_{9}$. We consider the eigenvalue problem

$$
K_{\mathrm{in}}^{D}(g) u=\tau u, \quad u \in H_{0}^{1}\left(G_{9}\right) \cap H^{2}\left(G_{9}\right) .
$$

We evaluate the number of eigenvalues lying in the interval $(k-g, k+g)$ of the above problem. Let $\left\{\tau_{j}\right\}, \tau_{j}=\tau_{j}(g)$, be the eigenvalues of (7.1) and let $\left\{u_{j}\right\}, u_{j}=u_{j}(x ; g)$, be the corresponding normalized eigenfunctions. Then the spectral function is defined as

$$
e(x, \tau ; g)=\sum_{\tau_{\jmath}<\tau} u_{\jmath}(x ; g) \bar{u}_{j}(x ; g)
$$

so that the number of eigenvalues less than $\tau$ is given by

$$
N\left(\tau ; K_{\text {in }}^{D}(g)\right)=\int_{G_{9}} e(x, \tau ; g) d x .
$$

We assert that

$$
N\left(\tau ; K_{\mathrm{in}}^{D}(g)\right)=c_{0} \tau+O\left(\tau^{1 / 2}\right), \quad \tau \rightarrow \infty,
$$

if the ratio $\kappa=\tau / g^{2}$ ranges over a compact interval $\Sigma=\left[\kappa_{1}, \kappa_{2}\right], 0<\kappa_{1}<$ $\kappa_{2}<\infty$, where

$$
\left.c_{0}=(2 \pi)^{-2} \operatorname{vol}\left\{(x, \xi): x \in G_{9}, \quad K(x, \xi ; g)<1\right)\right\}=(2 \pi)^{-1} \operatorname{meas}\left(G_{9}\right)
$$

and the remainder estimate is uniform in $g \gg 1$ and $\kappa \in \Sigma$. We first complete the proof of Lemma 5.2, accepting (7.2) as proved.

Proof of Lemma 5.2. Recall the definition (5.8) of $F_{2}$

$$
F_{2}(k+i \epsilon ; g)=I d-\psi_{2} W(g) R\left(k+i \epsilon ; K^{D}(g)\right) \psi_{2},
$$


where the coefficients of first order differential operator $W(g)$ have support in $G_{4}$ and obey the bound $O\left(g^{2}\right)$. Hence the second operator on the right side can be regarded as an operator from $L^{2}\left(G_{9}\right)$ into itself. We set

$$
T(k+i \epsilon ; g)=\psi_{2} W(g) R\left(k+i \epsilon ; K_{\mathrm{in}}^{D}(g)\right): L^{2}\left(G_{9}\right) \rightarrow L^{2}\left(G_{9}\right) .
$$

Let $E(g)$ denote the spectral resolution over the interval $(k-g, k+g)$ of $K_{\text {in }}^{D}(g)$. We now use (7.2) with $\tau=k \pm g \sim g^{2}$. Then it follows that

$$
m(g)=\operatorname{dim} \operatorname{Ran} T(k+i \epsilon ; g) E(g)=O(g), \quad g \rightarrow \infty .
$$

unifomly in $\epsilon$. As is easily seen,

$$
\nabla\left(K_{\mathrm{in}}^{D}(g)+1\right)^{-1}: L^{2}\left(G_{9}\right) \rightarrow L^{2}\left(G_{9}\right)
$$

is bounded with bound $O(g)$ and hence $W(g)\left(K_{\text {in }}^{D}(g)+1\right)^{-1}$ is also bounded with bound $O\left(g^{3}\right)$. Thus we have that

$$
T(k+i \epsilon ; g)(I d-E(g)): L^{2}\left(G_{9}\right) \rightarrow L^{2}\left(G_{9}\right)
$$

obeys the bound $O\left(g^{4}\right)$ uniformly in $\epsilon$. We now assume that $n>M g>m(g)$ for some $M \gg 1$ and use Proposition 3.1 to obtain that

$$
s_{n}\left(F_{2}(k+i \epsilon ; g)\right) \leq s_{1}(I d)+\left\|T(k+i \epsilon ; g)(I d-E(g)) \psi_{2}\right\|=O\left(g^{4}\right)
$$

uniformly in $\epsilon$. This proves the lemma.

7.2. We come back to (7.2). This formula is a more or less well known result. We give only a sketch for a proof. We divide $G_{9}$ into three regions $G_{9}=\cup \Omega_{j}, 0 \leq j \leq 2$, where $\Omega_{0}=\{x:|x|<8\}$ and

$$
\Omega_{1}=\left\{x: 7<|x|<9-1 / \tau^{1 / 2}\right\}, \quad \Omega_{2}=\left\{x: 9-2 / \tau^{1 / 2}<|x|<9\right\} .
$$

Let $\left\{\varphi_{0}, \varphi_{1}, \varphi_{2}\right\}$ be a smooth partition of unity on $G_{9}$ subject to this division and normalized by

$$
\varphi_{0}(x)^{2}+\varphi_{1}(x ; \tau)^{2}+\varphi_{2}(x ; \tau)^{2}=1 .
$$

We write $\exp \left(-t K_{\text {in }}^{D}(g)\right)(x, y), t \geq 0$, for the Green kernel of the semigroup $\exp \left(-t K_{\text {in }}^{D}(g)\right)$. Then we have

$$
\left|\exp \left(-t K_{\text {in }}^{D}(g)\right)(x, x)\right| \leq \exp \left(-t H_{0}\right)(x, x)=O\left(t^{-1}\right), \quad t \rightarrow 0
$$


by the Feynman-Kac-Ito formula $([3])$. This implies that $e(x, \tau ; g)=O(\tau)$ uniformly in $g$ and hence

$$
\int_{G_{9}} \varphi_{2}(x ; \tau)^{2} e(x, \tau ; g) d x=O\left(\tau^{1 / 2}\right) .
$$

According to definition $(5.2), K_{\text {in }}^{D}(g)$ acts as $(-i \nabla-g \beta \nabla \gamma)^{2} / 2$ near the boundary $\partial G_{9}$. Since $\exp (i[g \beta] \gamma(x))$ is smooth in $\Omega_{1}$, this operator is transformed to $(-i \nabla-(g \beta-[g \beta]) \nabla \gamma)^{2} / 2$ by the gauge transformation, which has smooth coefficients bounded uniformly in $g \gg 1$ in the region $\Omega_{1}$. Hence we can apply the same argument as in [16] to obtain that

$$
\int_{G_{9}} \varphi_{1}(x ; \tau)^{2} e(x, \tau ; g) d x=(2 \pi)^{-1} \int_{G_{9}} \varphi_{1}(x ; \tau)^{2} d x \tau+O\left(\tau^{1 / 2}\right) .
$$

On the other hand, the problem in the interior domain $\Omega_{0}$ is treated as the semi-classical problem on the number of eigenvalues less than $\kappa=\tau / g^{2}$, $\kappa \in \Sigma$ being fixed, of the operator $\left(-i h \nabla-A_{1}\right)^{2} / 2, h=g^{-1}$. We can neglect a contribution from the boundary $\partial G_{9}$ and it essentially becomes the problem over the whole space $R^{2}$. We use the method standard in the semi-classical spectral analysis (for example, see [13]) to obtain that

$$
\int_{G_{9}} \varphi_{0}(x)^{2} e(x, \tau ; g) d x=(2 \pi)^{-1} \int_{G_{9}} \varphi_{0}(x)^{2} d x \tau+O\left(\tau^{1 / 2}\right),
$$

which, together with (7.3) and (7.4), proves (7.2).

\section{$\S 8$. Proof of Lemma 5.3}

We here prove the last lemma (Lemma 5.3). The proof uses a simple theory of ordinary differential equations.

8.1. We first recall the definition (5.9) of $Y(\mu ; g), \mu=k+i \epsilon$. It contains the difference between the resolvents $R\left(\mu ; K_{\beta}(g)\right)$ and $R\left(\mu ; K_{\beta}^{D}(g)\right)$. Both the resolvents have the polar coordinate decomposition (see subsection $6.2)$. Thus we consider the operator

$$
\begin{aligned}
\Gamma_{l}(\mu ; g)=\psi_{2}\left\{R\left(\mu ; P_{\beta l}(g)\right)-R\left(\mu ; P_{\beta l}^{D}(g)\right)\right\} \chi_{9}: L^{2}((0, \infty) & ; d r) \\
& \longrightarrow L^{2}((0, \infty) ; d r)
\end{aligned}
$$

where $\psi_{2}(x)=\psi\left(|x| / 2^{2}\right)$ is regarded as a function of $r=|x|$ and $\chi_{9}=\chi_{9}(r)$ is again the characteristic function of the interval $[0,9]$. If $|l|>L_{0} g$ with some $L_{0} \gg 1$, then there exists the limit

$$
\psi_{2} R\left(k+i 0 ; P_{\beta l}^{D}(g)\right) \chi_{9}=\lim _{\epsilon \rightarrow 0} \psi_{2} R\left(k+i \epsilon ; P_{\beta l}^{D}(g)\right) \chi_{9}
$$


in norm as a bounded operator on $L^{2}((0, \infty) ; d r)$ (see Lemma 6.4). On the other hand, the resolvent $R\left(k+i \epsilon ; K_{\beta}(g)\right)$ has also the limit

$$
\psi_{2} R\left(k+i 0 ; K_{\beta}(g)\right) \chi_{9}=\lim _{\epsilon \rightarrow 0} \psi_{2} R\left(k+i \epsilon ; K_{\beta}(g)\right) \chi_{9}: L^{2}\left(R^{2}\right) \rightarrow L^{2}\left(R^{2}\right)
$$

by the principle of limiting absorption and hence $\psi_{2} R\left(k+i \epsilon ; P_{\beta l}(g)\right) \chi_{9}$ converges in norm as a bounded operator on $L^{2}((0, \infty) ; d r)$ uniformly in $l \in Z$. Thus we can define

$$
\Gamma_{l}(k ; g)=\lim _{\epsilon \rightarrow 0} \Gamma_{l}(k+i \epsilon ; g): L^{2}((0, \infty) ; d r) \rightarrow L^{2}((0, \infty) ; d r)
$$

for $|l|>L_{0} g$, where the convergence is uniform in $l$.

8.2. Let $L_{0} \gg 1$ be as above. We now define $v_{l}=v_{l}(r ; k, g),|l|>L_{0} g$, as a real solution to $P_{\beta l}(g) v_{l}=k v_{l}$ in $(0, \infty)$ with normalization

$$
\int_{0}^{9}\left|v_{l}(r ; k, g)\right|^{2} d r=1 .
$$

In other words, $v_{l}$ solves the equation

$$
-v_{l}^{\prime \prime}+q_{l}(r ; g) v_{l}=2 k v_{l} \quad \in(0, \infty)
$$

where

$$
q_{l}(r ; g)=\left(\left(l-g \beta \psi_{\infty}(r)\right)^{2}-1 / 4\right) r^{-2}+2 N g^{2} \psi_{1}(r) .
$$

Such a solution exists uniquely except for the sign \pm . We define $I_{l}$ as

$$
I_{l} u=\left(u, \chi_{9} v_{l}\right)_{r} \chi_{9} v_{l}: L^{2}((0, \infty) ; d r) \rightarrow L^{2}((0, \infty) ; d r),
$$

where $(,)_{r}$ denotes the $L^{2}$ scalar product in $L^{2}((0, \infty) ; d r)$.

LEMmA 8.1. Let $v_{l},|l|>L_{0} g$, be as above. Then one has

$$
\int_{0}^{8}\left|v_{l}(r ; k, g)\right|^{2} d r=O\left(|l|^{-d}\right)
$$

for any $d \gg 1$ and, in particular, $I_{l} \psi_{2}$ obeys the bound $O\left(|l|^{-d}\right)$ as a bounded operator on $L^{2}((0, \infty) ; d r)$. 
Proof. Let $\varphi \in C_{0}^{\infty}([0,9))$ be a non-negative function with compact support. We take the scalar product in $L^{2}((0, \infty) ; d r)$ of $\varphi^{2} v_{l}$ with equation (8.1). Since $q_{l}(r ; g) \geq C l^{2} \gg k$ for $r \in(0,9)$, we integrate the resulting relation by parts to obtain that

$$
\left(\varphi v_{l}, \varphi v_{l}\right)_{r} \leq C l^{-2}\left\{\left(\varphi^{\prime} v_{l}, \varphi^{\prime} v_{l}\right)_{r}+\left|\left(\varphi^{\prime \prime} v_{l}, \varphi v_{l}\right)_{r}\right|\right\}=O\left(l^{-2}\right) .
$$

The lemma is proved by repeated use of this inequality.

Lemma 8.2. Assume that $|l|>L_{0} g$ for $L_{0} \gg 1$ as above. Let $\Gamma_{l}(k ; g)$ and $I_{l}$ be defined as above. Then one has

$$
\Gamma_{l}(k ; g) \psi_{2}=\Gamma_{l}(k ; g) I_{l} \psi_{2}
$$

as an operator on $L^{2}((0, \infty) ; d r)$.

Proof. Let $u_{l}=\psi_{2} u-I_{l} \psi_{2} u$ for $u \in L^{2}((0, \infty) ; d r)$. Then $u_{l}$ has support in $[0,9]$ and it follows by definition that

$$
\left(u_{l}, \chi_{9} v_{l}\right)_{r}=0 .
$$

To prove the lemma, it suffices to show that

$$
\Gamma_{l}(k ; g) u_{l}=0 \text {. }
$$

Let $G_{l}\left(r, r^{\prime}\right)$ be the Green function of $R\left(k+i 0 ; P_{\beta l}(g)\right)$. It takes the form $G_{l}\left(r, r^{\prime}\right)=f_{l}\left(r_{-}\right) h_{l}\left(r_{+}\right)$, where $r_{-}=\min \left(r, r^{\prime}\right)$ and $r_{+}=\max \left(r, r^{\prime}\right)$. The functions $f_{l}$ and $h_{l}$ are linearly independent solutions to $\left(P_{\beta l}(g)-k\right) w=0$ in $(0, \infty)$. The function $f_{l}$ concides with $v_{l}$ and is bounded near the origin $r=0$. On the other hand, $h_{l}$ coincides with $c r^{1 / 2} H_{\nu}^{(1)}(\sqrt{2 k} r), \nu=|l-g \beta|$, for $r>9$ and satisfies the outgoing radiation condition at infinity, where $H_{\nu}^{(1)}$ is the Hankel function and the constant $c, c=c(k, g)$, is chosen in such a way that the Wronskian for pair of solutions $\left(f_{l}, h_{l}\right)$ equals one. By (8.3), we have

$$
\left(R\left(k+i 0 ; P_{\beta l}(g)\right) u_{l}\right)(r)=h_{l}(r) \int_{0}^{r} v_{l}\left(r^{\prime}\right) u_{l}\left(r^{\prime}\right) d r^{\prime}=0 \quad \text { at } r=9 .
$$

This shows that

$$
R\left(k+i 0 ; P_{\beta l}(g)\right) u_{l}=R\left(k+i 0 ; P_{\beta l}^{D}(g)\right) u_{l}
$$

and hence (8.4) is obtained. 
LEMMA 8.3. Assume again that $|l|>L_{0} g$. Let

$$
P_{0 l}=\left(-\partial_{r}^{2}+\left(l^{2}-1 / 4\right) r^{-2}\right) / 2
$$

be the operator obtained from the free Hamiltonian $H_{0}=-\Delta / 2$ by the expansion in angular momentum channels. Then one has

$$
\left(P_{0 l}+I d\right)^{1 / 2} \Gamma_{l}(k ; g): L^{2}((0, \infty) ; d r) \rightarrow L^{2}((0, \infty) ; d r)
$$

is bounded and obeys the bound $O\left(l^{-1}\right)$.

This lemma can be easily verified by use of Lemmas 6.3 and 6.4 . We skip the proof. We now combine the three lemmas above to obtain that for any $d \gg 1$

$$
\left\|\left(P_{0 l}+I d\right)^{1 / 2} \Gamma_{l}(k ; g) \psi_{2}\right\|=O\left(g^{-d}\right), \quad g \rightarrow \infty
$$

uniformly in $|l|>L_{0} g$ as a bounded operator on $L^{2}((0, \infty) ; d r)$. Since

$$
\left(P_{0 l}+I d\right)^{1 / 2} \Gamma_{l}(k+i \epsilon ; g) \rightarrow\left(P_{0 l}+I d\right)^{1 / 2} \Gamma_{l}(k ; g), \quad \epsilon \rightarrow 0,
$$

converges in norm uniformly in $|l|>L_{0} g$, it follows that: For given $d \gg 1$, there exists $\epsilon(d, g)>0$ such that

$$
\left\|\left(P_{0 l}+I d\right)^{1 / 2} \Gamma_{l}(k+i \epsilon ; g) \psi_{2}\right\| \leq C g^{-d}, \quad g \gg 1
$$

for $0<\epsilon<\epsilon(d, g)$ and $l$ as above.

8.3. We are now in a position to prove Lemma 5.3.

Proof of Lemma 5.3. We recall the definition (5.9) of $Y(k+i \epsilon ; g)$. The operator has the composition

$$
Y(k+i \epsilon ; g)=W(g)\left(H_{0}+I d\right)^{-1 / 2} F_{4}(k+i \epsilon ; g) F_{2}(k+i \epsilon ; g),
$$

where $F_{2}$ is defined by (5.8) and

$$
F_{4}(k+i \epsilon ; g)=\left(H_{0}+I d\right)^{1 / 2} \psi_{2}\left\{R\left(k+i \epsilon ; K_{\beta}(g)\right)-R\left(k+i \epsilon ; K_{\beta}^{D}(g)\right)\right\} \psi_{2} .
$$

It has been already shown (Lemma 5.2) that

$$
\limsup _{\epsilon \rightarrow 0} s_{n}\left(F_{2}(k+i \epsilon ; g)\right) \leq C g^{4}
$$


for $n>M_{1} g$ with some $M_{1} \gg 1$ and also we have

$$
s_{1}\left(W(g)\left(H_{0}+I d\right)^{-1 / 2}\right) \leq C g^{2} .
$$

We assert that there exist $M \gg 1$ such that

$$
\limsup _{\epsilon \rightarrow 0} s_{n}\left(F_{4}(k+i \epsilon ; g)\right) \leq C g^{-d}, \quad d \gg 1,
$$

for $n>M g$. If this is verified, then it follows from Proposition 3.1 that

$$
\limsup _{\epsilon \rightarrow 0} s_{n}(Y(k+i \epsilon ; g))<1 / 2, \quad g \gg 1,
$$

for $n>M g$ with another $M \gg 1$ and hence we have

$$
s_{n}(Y(k+i \epsilon ; g)) \leq 1 / 2, \quad n>M g,
$$

for $0<\epsilon<\epsilon(n, g)$ with some $\epsilon(n, g)$ depending on $n$ and $g$. This, together with Proposition 3.1 again, implies that $s_{n}\left(F_{3}(k+i \epsilon ; g)\right) \leq 2$ for $\epsilon$ as above and the lemma follows at once.

We shall prove the above assertion (8.6). Let $\mathcal{U}$ be the unitary mapping defined by (2.6) and let

$$
Q_{l}: L^{2}((0, \infty) ; d r) \otimes L^{2}\left(S^{1}\right) \rightarrow L^{2}((0, \infty) ; d r) \otimes \Lambda_{l}
$$

be a projection, where $\Lambda_{l}$ is again the eigenspace of $-i \partial / \partial \theta$ associated with eigenvalue $l$. Define

$$
F_{4, n}(k+i \epsilon ; g)=F_{4}(k+i \epsilon ; g) \mathcal{U}^{-1}\left[\sum_{|l|<[n / 2]} \oplus Q_{l}\right] \mathcal{U}: L^{2}\left(R^{2}\right) \rightarrow L^{2}\left(R^{2}\right)
$$

As is easily seen,

$$
\operatorname{dim} \operatorname{Ran}\left\{R\left(k+i \epsilon ; P_{\beta l}(g)\right)-R\left(k+i \epsilon ; P_{\beta l}^{D}(g)\right)\right\} \leq 2
$$

as an operator from $L^{2}((0, \infty) ; d r)$ into itself and hence

$$
\operatorname{dim} \operatorname{Ran} F_{4, n}(k+i \epsilon ; g)<n .
$$

We now assume that $n>2 L_{0} g+2$. Then it follows from (8.5) that $\left\|F_{4}(k+i \epsilon ; g)-F_{4, n}(k+i \epsilon ; g)\right\| \leq \max _{|l|>L_{0} g}\left\|\left(P_{0 l}+I d\right)^{1 / 2} \Gamma_{l}(k+i \epsilon ; g) \psi_{2}\right\| \leq C g^{-d}$ for $d \gg 1$ and $\epsilon>0$ as in (8.5). This proves (8.6) and the proof is complete. 


\section{$\S 9$. Sacttering by magnetic fields with small support}

We here study the cross section for scattering by magnetic fields with small support. Throughout the section, we fix $h=1$ and again assume the magnetic field $b(x) \in C_{0}^{\infty}\left(R^{2}\right)$ to be supported in the unit ball $G_{1}$, $\operatorname{supp} b \subset G_{1}$.

We consider the magnetic field

$$
b_{R}(x)=R^{-2} b(x / R), \quad 0<R \ll 1,
$$

with support in $G_{R}$. As is easily seen, $b_{R}$ conserves the flux

$$
\int b_{R} d x=\int b d x=2 \pi \beta
$$

The corresponding megnetic potential $A_{R}(x)$ is given by $A_{R}=R^{-1} A(x / R)$, $d A_{R}=b_{R} d x_{1} \wedge d x_{2}$, where $A$ is still defined by (1.4). We denote by $H\left(A_{R}\right)$ the Hamiltonian with $A_{R}$ as a magnetic potential and by $\sigma\left(\lambda, \omega ; b_{R}\right)$ the quantity defined by (1.11) for pair $\left(H_{0}, H\left(A_{R}\right)\right)$. We also denote by $\sigma_{\text {tot }}\left(\lambda, \omega ; b_{R}\right)$ the total cross section for integer $\beta \in Z$.

We now consider the integral

$$
\int_{I} \sigma\left(\lambda, \omega ; b_{R}\right) d \lambda
$$

for compact interval $I=[c, d], 0<c<d<\infty$, fixed. The aim here is to study the bound of this integral as $R \rightarrow 0$ and the special emphasis is placed on the dependence on the value $\beta$.

9.1 We shall formulate the main result obtained here. Let

$$
\alpha=1 \text { for } \beta \in Z, \quad \alpha=\min \{\nu=|l-\beta| ; l \in Z\} \text { for } \beta \notin Z \text {. }
$$

If we write $\beta=[\beta]+\kappa, 0<\kappa<1$, for $\beta \notin Z$, then $\alpha=\min (\kappa, 1-\kappa)$.

THEOREM 9.1. Let the notations be as above. Then one has

$$
\int_{I} \sigma\left(\lambda, \omega ; b_{R}\right) d \lambda=O\left(R^{2 \alpha-2}\right), \quad R \rightarrow 0
$$

and if, in particular $\beta \in Z$, then

$$
\int_{I} \sigma_{\text {tot }}\left(\lambda, \omega ; b_{R}\right) d \lambda=O(1), \quad R \rightarrow 0 .
$$


We shall give a simple application of the above theorem. Let $\mid f(\omega \rightarrow$ $\left.\theta, \lambda ; b_{R}\right)\left.\right|^{2}$ be the differential cross section for pair $\left(H_{0}, H\left(A_{R}\right)\right)$ and let $C=$ $(0, \infty) \times \Lambda$ be the cone generated by the compact subset $\Lambda \subset S^{1}$, where $\Lambda$ does not contain the incident direction $\omega$. Then the integral

$$
\int_{\Lambda}\left|f\left(\omega \rightarrow \theta, \lambda ; b_{R}\right)\right|^{2} d \theta
$$

is finite even for $\beta \notin Z$ and describes the total number of particles scattered into cone $C$ at energy $\lambda$. The following result is obtained as an immdiate consequence of Theorem 9.1 .

\section{Corollary 9.2.}

$$
\int_{I} \int_{\Lambda}\left|f\left(\omega \rightarrow \theta, \lambda ; b_{R}\right)\right|^{2} d \theta d \lambda=O\left(R^{2 \alpha-2}\right), \quad R \rightarrow 0 .
$$

Remark. Theorem 9.1 should be compared with the bound obtained by Enss-Simon [4] in the case of potential scattering. Let $V_{R}(x), x \in R^{2}$, be a finite-range potential with support in $G_{R}$ and let $\sigma_{\text {tot }}\left(\lambda, \omega ; V_{R}\right)$ be the total cross section for the Schrödinger operator $H_{0}+V_{R}$. Then the following bound has been obtained in [4] (Theorems 3.1 and 3.2):

$$
\int_{I} \sigma_{\mathrm{tot}}\left(\lambda, \omega ; V_{R}\right) d \lambda=O\left(R^{-2}\right), \quad R \rightarrow 0
$$

and if, in particular, $V_{R} \geq 0$, then the bound above is of order $O(1)$, where the order relations are independent of $V_{R}$. The corresponding bound for the magnetic scattering case seems to depend strongly on the flux of magnetic fields.

9.2. We shall prove Theorem 9.1. The first step toward the proof is to show the following

LEMMA 9.3. One has the relation

$$
\sigma\left(\lambda, \omega ; b_{R}\right)=R \sigma\left(R^{2} \lambda, \omega ; b\right)
$$

The lemma may be verified by making a change of variables. However we give an alternative proof. The proof is based on the lemma below, which has been already obtained as Lemma 5.3 of [19]. 
Lemma 9.4. Let $F_{\omega}(y), y=\langle x, \omega\rangle$, be defined by

$$
F_{\omega}(y)=(2 \pi)^{-1 / 2} \int \exp (i \sqrt{2 \lambda} y)(2 \lambda)^{-1 / 4} F(\lambda) d \lambda
$$

for $F \in C_{0}^{\infty}((0, \infty))$. Let $\phi_{L}=\psi(|z| / L), L \gg 1$, where $\psi$ is as in $(1.14)$ and $z$ is in the straight line (impact plane) $\Pi_{\omega}$ perpendicular to direction $\omega$. Write $S$ and $S_{\beta}$ for the scattering operators $S\left(H, H_{0}\right), H=H(A)$, and $S\left(H_{\beta}, H_{0}\right)$, respectively. Then the limit

$$
\left\|\left(S-S_{\beta}\right) F_{\omega}\right\| \equiv \lim _{L \rightarrow \infty}\left\|\left(S-S_{\beta}\right) \phi_{L} F_{\omega}\right\|
$$

exists, where the limit is denoted as the term on the left side, and one has

$$
\left\|\left(S-S_{\beta}\right) F_{\omega}\right\|^{2}=\int|F(\lambda)|^{2} \sigma(\lambda, \omega ; b) d \lambda .
$$

Proof of Lemma 9.3. The quantities on both sides of the relation in the lemma are continuous in $\lambda>0$. Hence it suffices to prove the lemma in the weak form. The lemma is verified in almost the same way as in the proof of Theorem A.1.1 of [4]. We give only a sketch for the proof.

Let

$$
\left(U_{R} f\right)(x)=R^{-1} f(x / R): L^{2}\left(R^{2}\right) \rightarrow L^{2}\left(R^{2}\right)
$$

be a unitary operator. Then a simple computation yields

$$
U_{R} H_{0} U_{R}^{-1}=R^{2} H_{0}, \quad U_{R} H U_{R}^{-1}=U_{R} H(A) U_{R}^{-1}=R^{2} H\left(A_{R}\right) .
$$

Hence it follows that $U_{R} S U_{R}^{-1}=S\left(H\left(A_{R}\right), H_{0}\right)$ for $S=S\left(H, H_{0}\right)$. We can also show that $U_{R} S_{\beta} U_{R}^{-1}=S_{\beta}$ for $S_{\beta}=S\left(H_{\beta}, H_{0}\right)$. Thus we use the relation (9.3) to obtain that

$$
\int|F(\lambda)|^{2} \sigma\left(\lambda, \omega ; b_{R}\right) d \lambda=\left\|\left(S-S_{\beta}\right) U_{R}^{-1} F_{\omega}\right\|^{2}
$$

for any $F \in C_{0}^{\infty}((0, \infty))$. The function $\left(U_{R}^{-1} F_{\omega}\right)(y)=R F_{\omega}(R y)$ on the right side is represented by integral (9.2) with $F$ replaced by $R^{-1 / 2} F\left(R^{-2} \lambda\right)$. This yields

$$
\int|F(\lambda)|^{2} \sigma\left(\lambda, \omega ; b_{R}\right) d \lambda=R \int|F(\lambda)|^{2} \sigma\left(R^{2} \lambda, \omega ; b\right) d \lambda
$$

by making a simple change of variable and the proof is complete. 
Let $F \in C_{0}^{\infty}((0, \infty))$ again. We define

$$
\sigma_{F}(R)=\int F(\lambda) \sigma\left(\lambda, \omega ; b_{R}\right) d \lambda
$$

Then it follows from Lemma 9.3 that

$$
\sigma_{F}(R)=R^{-1} \int F\left(R^{-2} \lambda\right) \sigma(\lambda, \omega ; b) d \lambda .
$$

We evaluate the integral on the left side. If, in particular, we take $F(\lambda) \geq 0$ with $F=1$ on $I$, then

$$
\int_{I} \sigma\left(\lambda, \omega ; b_{R}\right) d \lambda \leq \sigma_{F}(R)
$$

9.3. We recall that $V=J_{a}\left[\psi_{0}, H_{0}\right]$ (see (2.5)), where $J_{a}$ is the multiplication operator with $\exp (i a(x))$ and $a(x)$ is defined by $(2.1)$. We note that $V$ has only the radial differentiation $\partial / \partial|x|$ as a first differential operator and its coefficients are supported in $G=\{x: 1<|x|<2\}$. We can represent $\sigma(\lambda, \omega ; b)$ as

$$
\sigma(\lambda, \omega ; b)=2 \pi(2 \lambda)^{-1 / 2}\left(E^{\prime}(\lambda) V \varphi_{-}(\lambda, \omega), V \varphi_{-}(\lambda, \omega)\right),
$$

where $E(\lambda)$ is the spectral resolution associated with $H=H(A), E^{\prime}(\lambda)=$ $d E(\lambda)) / d \lambda$ and $\varphi_{-}(\lambda, \omega)=\varphi_{-}(x, \lambda, \omega)$ is the outgoing eigenfunction of $H_{\beta}=H(B), B=\beta \nabla \gamma(x)$, with eigenvalue $\lambda$ (see (2.10)). This relation can be obtained in the same way as used to prove Lemma 2.2 (see also Proposition 5.2 of [19]). Thus the quantity $\sigma_{F}(R)$ under consideration is now rewritten as

$$
\sigma_{F}(R)=2 \pi R^{-1} \int(2 \lambda)^{-1 / 2} F\left(R^{-2} \lambda\right)\left(E^{\prime}(\lambda) V \varphi_{-}(\lambda, \omega), V \varphi_{-}(\lambda, \omega)\right) d \lambda .
$$

We evaluate this integral by use of partial integration. We note that $\lambda \sim R^{2}$ when $\lambda$ is restricted to the support of function $F\left(R^{-2} \cdot\right)$.

9.4. We first prove the theorem for the case $\beta \notin Z$. We have to look at the behavior of $\varphi_{-}(x, \lambda, \omega), x \in G$, as $\lambda \rightarrow 0$, where $G=\{x: 1<|x|<2\}$ again. Let $\alpha$ be as in (9.1) and let $l^{*} \in Z$ be such that $\alpha=\left|l^{*}-\beta\right|$. According to definition (2.10), the main contribution comes from Bessel function $J_{\alpha}(\sqrt{2 \lambda}|x|)$ of order $\alpha$, and $\varphi_{-}$behaves like

$$
\varphi_{-}(x, \lambda, \omega)=C_{\alpha} \exp \left(i l^{*} \zeta(x)\right) \lambda^{\alpha / 2}|x|^{\alpha}+o\left(\lambda^{\alpha / 2}\right), \quad \lambda \rightarrow 0,
$$


for some constant $C_{\alpha}$ uniformly in $x \in G$, where $\zeta(x)=\gamma(x ;-\omega)$ is the azimuth angle from $-\omega$, so that $\partial \zeta(x) / \partial|x|=0$. The remainder estimate above remains true in the $C^{\infty}$ topology as a function of $x$. Thus the lemma below can be easily verified and the bound $O\left(R^{2 \alpha-2}\right)$ in Theorem 9.1 follows from this lemma by partial integration.

Lemma 9.5. Let $\alpha$ be as above. Then one has:

$$
\begin{aligned}
& \varphi_{-}(x, \lambda, \omega)=O\left(\lambda^{\alpha / 2}\right), \quad\left(\partial^{2} / \partial|x| \partial \lambda\right) \varphi_{-}(x, \lambda, \omega)=O\left(\lambda^{\alpha / 2-1}\right), \\
& (\partial / \partial|x|) \varphi_{-}(x, \lambda, \omega)=O\left(\lambda^{\alpha / 2}\right), \quad(\partial / \partial \lambda) \varphi_{-}(x, \lambda, \omega)=O\left(\lambda^{\alpha / 2-1}\right),
\end{aligned}
$$

where the order relation means that the $L^{2}$ norm in $G$ obeys the prescribed bound as $\lambda \rightarrow 0$.

Next we discuss the case $\beta \in Z$. We assume for a moment that $\beta=0$, so that $B(x)=\beta \nabla \gamma(x)=0$ and also

$$
\varphi_{-}(x, \lambda, \omega)=\varphi_{0}(x, \lambda, \omega)=\exp (i \sqrt{2 \lambda}\langle x, \omega\rangle) .
$$

As is easily seen,

$$
V \varphi_{-}=2^{-1} \exp (i a(x))\left(\Delta \psi_{0}\right) \varphi_{0}+O\left(\lambda^{1 / 2}\right),
$$

where the order relation is used with the same meaning as in Lemma 9.5. We can rewrite the leading term on the right side as

$$
2^{-1} \exp (i a(x))\left\langle\nabla, \varphi_{0} \nabla \psi_{0}\right\rangle+O\left(\lambda^{1 / 2}\right)
$$

Hence it can be further rewritten as

$$
2^{-1}\left\langle(\nabla-i \nabla a), \exp (i a(x)) \varphi_{0} \nabla \psi_{0}\right\rangle+O\left(\lambda^{1 / 2}\right)
$$

by a simple use of gauge transformation. According to relation (2.2), $A(x)$ $=\nabla a(x)$ on $G$. Since

$$
\|E(\lambda)(\nabla-i A)\|=\left\|E(\lambda)(H+\lambda)^{1 / 2}(H+\lambda)^{-1 / 2}(\nabla-i A)\right\|=O\left(\lambda^{1 / 2}\right)
$$

as a bounded operator on $L^{2}\left(R^{2}\right)$, we have

$$
\int\left|\left(E(\lambda) V \varphi_{-}\right)(x)\right|^{2} d x \leq C \lambda
$$


On the other hand, it is easy to see that $V(\partial / \partial \lambda) \varphi_{-}=O\left(\lambda^{-1 / 2}\right)$. Thus the desired bound $O(1)$ in Theorem 9.1 is obtained for the case $\beta=0$.

A similar argument applies to the general case $\beta \neq 0$ also. In this case,

$$
\varphi_{-}(x, \lambda, \omega)=\exp (i \beta \zeta(x)) \varphi_{0}(x, \lambda, \omega),
$$

which can be seen from definition (2.10). We should note that $\exp (i \beta \zeta(x))$ is smooth in $R^{2} \backslash\{0\}$ for $\beta \in Z$ and also it follows that $\beta \nabla \zeta(x)=\beta \nabla \gamma(x)=$ $B(x)$. Since $\partial \zeta(x) / \partial|x|=0$ as previously stated, we have

$$
V \varphi_{-}=\exp (i(a(x)+\beta \zeta(x)))\left[\psi_{0}, H_{0}\right] \varphi_{0} .
$$

This enables us to repeat the same arguemnt as above and we can obtain the desired bound $O(1)$ for the general case also. Thus the proof of Theorem 9.1 is now complete.

\section{REFERENCES}

[1] S. Agmon, Some new results in spectral and scattering theory of differential operators on $R^{n}$, Seminaire Goulaouic-Schwartz, 1978.

[2] W. O. Amrein, J. M. Jauch and K. B. Sinha, Scattering Theory in Quantum Mechanics, W. A. Benjamin. Inc., 1977.

[3] J. Avron, I. Herbst and B. Simon, Schrödinger operators with magnetic fields. I, General interactions, Duke Math. J., 45 (1978), 847-883.

[4] V. Enss and B. Simon, Finite total cross sections in nonrelativistic quantum mechanics, Commun. Math. Phys., 76 (1980), 177-209.

[5] A. Erdélyi, Higher Transcendental Functions, Vol. II, Robert E. Krieger Publ. Company Inc., 1953.

[6] C. Gérard and A. Martinez, Principe d'absorption limite pour des opérateurs de Schrödinger à longue portée, C. R. Acad. Sci. Paris, 306 (1988), 121-123.

[7] C. Gérard, A. Martinez and D. Robert, Breit-Wigner formulas for the scattering phase and the total scattering cross-section in the semı-classical limit, Commun. Math. Phys., 121 (1989), 323-336.

[8] I. C. Gohberg and M. G. Krein, Introduction to the theory of linear nonselfadjoint operators, Translations of Mathematical Monographs, Vol. 18, A. M. S., 1969.

[9] T. Ikebe and Y. Saitō, Limiting absorption method and absolute continuity for the Schrödinger operators, J. Math. Kyoto Univ., 7 (1972), 513-542.

[10] H. Isozaki and H. Kitada, Scattering matrices for two-body Schrödinger operators, Sci. Papers College Arts Sci. Univ. Tokyo, 35 (1985), 81-107.

[11] M. Loss and B. Thaller, Scattering of particles by long-range magnetic fields, Ann. of Phys., 176 (1987), 159-180.

[12] P. A. Perry, Scattering Theory by the Enss Method, Mathematical Reports 1, Harwood Academic, 1983. 
[13] D. Robert, Autour de l'approximation Semi-classique, Birkhäuser, 1987.

[14] D. Robert and H. Tamura, Semi-classical estimates for resolvents and asymptotics for total scattering cross-sections, Ann. Inst. Henri Poincaré, 46 (1987), 415-442.

[15] S. N. M. Ruijsenaars, The Aharonov-Bohm effect and scattering theory, Ann. of Phys., 146 (1983), 1-34.

[16] R. Seeley, An estrmate near the boundary for the spectral function of the Laplace operator, Amer. J. Math., 102 (1980), 869-902.

[17] A. V. Sobolev, On the total scattering cross section for a fintte-range potential, Leningrad Math. J., 1 (1990), 1015-1026.

[18] A. V. Sobolev and D. R. Yafaev, On the quasi-classical limit of the total scattering cross-section in nonrelativistic quantum mechanics, Ann. Inst. Henri Poincaré, 44 (1986), 195-210.

[19] H. Tamura, Semi-classical analysis for total cross sections of magnetic Schrödinger operators in two dimensions, Rev. Math. Phys., 7 (1995), 443-480.

[20] H. Tamura, Shadow scattering by magnetıc fields in two dimensions, Ann. Inst. Henri Poincaré, 63 (1995), 253-276.

Department of Mathematics

Ibaraki University

Mito, Ibaraki 310

Japanv

tamura@mito.ipc.ibaraki.ac.jp 